\title{
Taxonomy and biogeography of the coastal fishes of Juan Fernández Archipelago and Desventuradas Islands, Chile
}

\author{
Taxonomía y biogeografía de los peces costeros del Archipiélago de
} Juan Fernández y de las islas Desventuradas, Chile

\author{
Brian S. Dyer ${ }^{1}$ and Mark W. Westneat ${ }^{2}$ \\ ${ }^{1}$ Escuela de Recursos Naturales, Universidad del Mar, Angamos 680, Reñaca, Viña del Mar, Chile. brian.dyer@udelmar.cl \\ ${ }^{2}$ Curator of Zoology, Field Museum of Natural History, 1400 S Lakeshore Dr., Chicago, IL 60605-2496, USA
}

Resumen.- Las islas Desventuradas (San Félix y San Ambrosio) y del Archipiélago de Juan Fernández (Robinson Crusoe y Alejandro Selkirk) son islas oceánicas de origen volcánico ubicadas en el Pacífico Sudeste, frente a Chile. Sobre la base de material recolectado desde 1997 hasta la fecha, revisiones de colecciones de las expediciones del R/V Anton Bruun y actualizaciones taxonómicas, la fauna de peces litorales del Archipélago de Juan Fernández y las islas Desventuradas tienen un total de 52 especies, con 41 y 43 especies, respectivamente. Los peces costeros endémicos de Juan Fernández son solamente cinco $(12,2 \%)$ y tres son las especies registradas aquí como nuevas para las islas (Gymnothorax cf. obesus, Gnathophis sp., Suezichthys sp.). Las Desventuradas tienen solamente dos especies endémicas (4,6\%) y cuatro son los nuevos registros (Scorpaenodes englerti, Maxillicosta reticulata, Suezichthys sp., Aseraggodes bahamondei). El reducido número de especies endémicas para Juan Fernández comparado con trabajos previos (15 especies) se debe a que muchas resultaron ser endémicas de ambos grupos de islas ( 22 especies $=42,3 \%$ total peces litorales), apoyando la propuesta que ambos grupos de islas debieran formar una sola unidad biogeográfica. La extensión oriental de la región Indo Pacífico Oeste para incluir la cordillera de Nazca, las islas Desventuradas y Juan Fernández encuentra apoyo en este trabajo. La afinidad zoogeográfica entre las Desventuradas y las islas del Pacífico Oeste (Isla de Pascua hasta Australia) incluye 16 especies, agregando cinco nuevos registros a listas anteriores. Filogenias de tres géneros señalan un centro de origen en el Pacífico Oeste, con uno o más eventos de dispersión hacia las islas Desventuradas y Juan Fernández. Este patrón de dispersión puede repetirse en otros siete géneros de pocas especies y relativamente restringidas en distribución. Solo una filogenia (Odontesthes) propone un origen continental con una dispersión hacia el Oeste, sin embargo otros tres géneros parecen tener este mismo patrón de dispersión.

Palabras clave: Diversidad, endemismo, Pacífico Sudeste

\begin{abstract}
The Desventuradas Islands (San Félix and San Ambrosio) and Juan Fernández Archipelago (Robinson Crusoe and Alejandro Selkirk) are oceanic islands of volcanic origin located in the southeastern Pacific off Chile. Based on new material collected since 1997, revision of the R/V Anton Bruun expeditions collections, and a taxonomic update, the coastal fish fauna of Juan Fernández Archipelago and Desventuradas Islands found a total of 52 species, with 41 and 43 species, respectively. The number of coastal fish species recorded herein as endemic for Juan Fernández is only five $(12,2 \%)$ and three species are new records (Gymnothorax cf. obesus, Gnathophis sp., Suezichthys sp). The Desventuradas Islands have only two endemic species $(4,6 \%)$ and four species are new records (Scorpaenodes englerti, Maxillicosta reticulata, Suezichthys sp., Aseraggodes bahamondei). The reduced number of endemic species for the Juan Fernández Islands as compared with previous accounts ( 15 species) is the result that more species were found to be endemic to both islands groups ( 22 species $=42,3 \%$ all coastal fishes), suggesting these two island groups should be considered a single biogeographic unit. The eastern extension of the Indo West Pacific Region to include the Nazca Ridge, Desventuradas and Juan Fernández Islands is supported herein. The zoogeographical affinities between Desventuradas and western Pacific Islands (Easter Island to Australia), includes 16 species, adding five new species to previous lists. Relationships of three genera indicate a Western Pacific origin with one or more dispersal events to the Desventuradas and Juan Fernández Islands. Another seven genera, with relatively few species and restricted distribution, may have this same pattern. Only one phylogenetic study indicates a continental origin with a westward dispersal (Odontesthes), however another three genera may have this same pattern of dispersal.
\end{abstract}

Key words: Diversity, endemism, southeastern Pacific 


\section{INTRODUCTION}

The fish fauna of the southeastern Pacific off Chile is a composite of subtropical eastern and western Pacific, southwestern Atlantic, and southern Australia-New Zealand subantarctic faunas (Pequeño 2000). Especially interesting zoogeographically are two island groups (Fig. 1), the northern one located about $850 \mathrm{~km}$ West of Chañaral (Desventuradas Islands: San Félix Is. $26^{\circ} 15^{\prime} \mathrm{S}, 80^{\circ} \mathrm{W}$; San Ambrosio Is. $26^{\circ} 20^{\prime} \mathrm{S}, 79^{\circ} 55^{\prime} \mathrm{W}$ ) and the southern one about $600 \mathrm{~km}$ West of Valparaíso (Juan Fernández Archipelago:

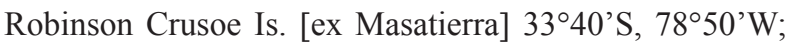

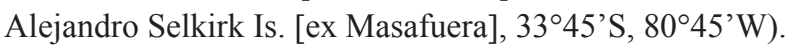
Juan Fernández Archipelago was declared National Park in 1935 and Reserve of the Biosphere in 1977.
The volcanic origin of these islands along "hot-spot lines" of the Nazca Plate are of Plio-Pleistocene age (González-Ferrán 1987). Potassium-argon dating of San Ambrosio Island gives a date of nearly 3 million years ago (m.y.a.) (González-Ferrán 1987:50) whereas Juan Fernández Islands range between 1-4 m.y.a. (Stuessy et al. 1984). The Desventuradas are the eastern outcrop of the "Easter hot line" that is connected by submarine seamouts to Easter Island and Salas y Gómez Island. The Juan Fernández Ridge extends from Alejandro Selkirk Island to the more eastern O'Higgins seamount, 60 nautical miles west of Valparaíso. The recent emergence of these islands does not impose a time limit on the connectivity of fish fauna for these islands as it does with the terrestrial

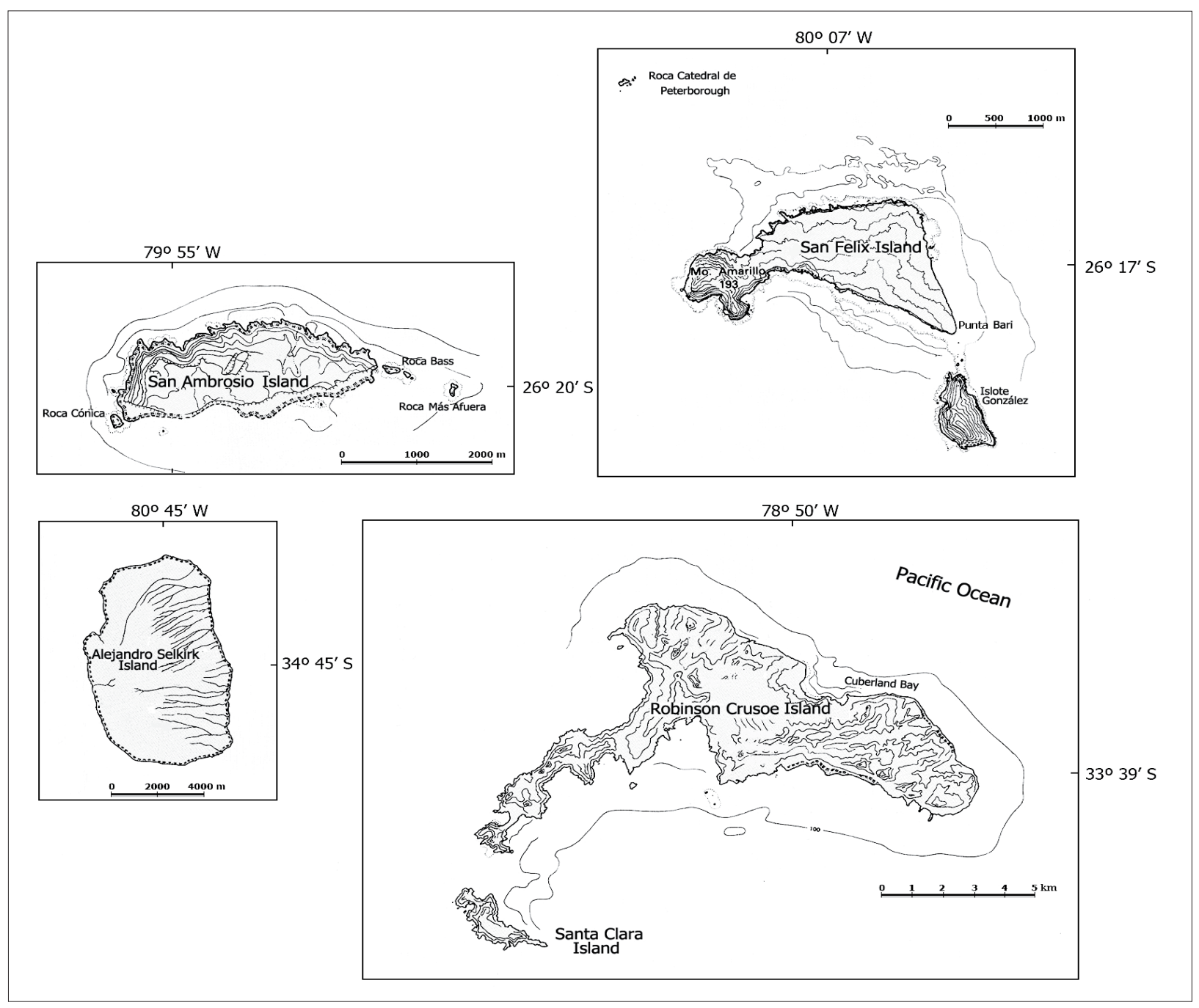

Figure 1. Map of the Chilean oceanic islands of Desventuradas and Juan Fernández Archipelago (SHOA 1994) / Mapa de las islas oceánicas chilenas Desventuradas y del Archipiélago de Juan Fernández (SHOA 1994) 


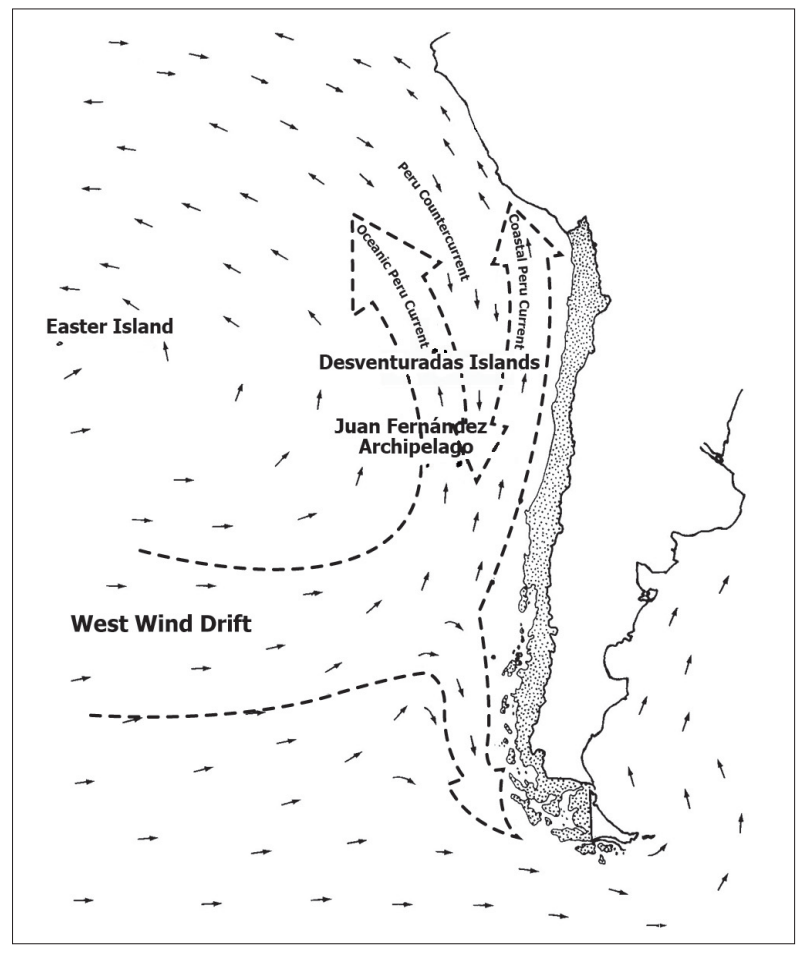

Figure 2. Map of the major currents affecting the oceanic islands of Desventuradas and Juan Fernández. Modified from Arana (1987:330) / Mapa de las principales corrientes que afectan las islas oceánicas de Juan Fernández y Desventuradas. Modificado de Arana (1987:330)

flora and fauna, but instead may be a key element in the historical perspective to the biogeographical interpretation of the faunal composition of these island groups.

The oceanographic regime of these islands is also of biogeographic interest. Both island groups are located between the coastal and oceanic branches of the subantarctic Perú or Humboldt Current which is split by the subtropical Peruvian countercurrent (Fig. 2). The surface temperatures are not subantarctic however because the sub-surface Peruvian countercurrent extends farther south than Juan Fernández and warms the surface waters notably between spring and autumn (Arana 1987). This Peruvian countercurrent is a possible migration route between these islands, either by adults or during a pelagic larval phase.

The Juan Fernández Islands have been surveyed substantially more often than the Desventuradas, most probably because of their proximity to Valparaíso and San Antonio, Chile's main ports. Most natural history studies show Juan Fernández to have a relatively high degree of endemism: 60-70\% vascular plants (Hoffman \& Marticorena 1987, Stuessy et al. 1992), 29-32\% algae
(Santelices 1987, 1992), 60-70\% arthropods (Peña 1987), $71 \%$ mollusks and $18 \%$ decapods (Roszbaczylo \& Castilla 1987). The Desventuradas Islands, on the other hand, are not along any navigational route, hence scientists have visited them only sporadically and for a short period of time allowing for only a few small collections. Endemism in Desventuradas is lower in general: one species of algae (7\%; Meneses \& Hoffmann 1994), 17 species of arthropods (16\%; Peña 1987), 14 species of terrestrial plants (74\%; Hoffmann \& Marticorena 1987).

The Desventuradas have had only three proper fishcollecting expeditions involving ichthyologists: the Anton Bruun cruises 12 (Wisner 1966) and 13 (Cornell Collection), and Pequeño \& Lamilla (2000). Wisner (1966:4) originally estimated a total of 27 fish species collected, though this number has actually increased to 31 species according to the holdings at Scripps Institute of Oceanography (SIO) (Pequeño \& Lamilla 2000). Pequeño \& Lamilla (2000) latest count of coastal fishes was 43 species, of which three are endemic to the Desventuradas Islands and $28(65 \%)$ are found also in Juan Fernández. Sepúlveda (1987) emphasized the fish fauna of the Juan Fernández Islands had not been studied as a whole since Skottsberg's Swedish expedition of 1908. About 140 fish species were recorded for the area around the Juan Fernández Archipelago, of which 58 were coastal species and eleven (19\%) endemic (Sepúlveda \& Pequeño 1985, Sepúlveda 1987, Meléndez \& Villalba 1992). The latest revision of the Juan Fernández coastal fish fauna notes 51 species (Pequeño \& Sáez 2000). However, 14 species are endemic to Juan Fernández Islands $(27 \%$ of the coastal fish fauna) and Desventuradas Islands (49\% of the coastal fish fauna). Taken both island groups together as a biogeographical unit, the combined endemism is of roughly $40 \%$. These endemics are congeneric with fishes from the Pacific Plate, Australia-New Zealand, or continental South America.

The main purpose of this paper was to present an updated faunal list of the coastal fishes of the Juan Fernández and Desventuradas Islands resulting from an expedition funded by National Geographic Society in 1997, to publish the first color photographs of most of these fishes, and comment on the biogeographic implications of their distributions. A better understanding of the aquatic flora and fauna, and their seasonal variation, will provide a more solid basis for a rational exploitation of the fishery resources already in practice around these islands (fishes and lobsters), contribute to the development of alternative income sources such as ecotourism (Cuevas 
Amphichaetodon melbae Antennarius coccineus Antennarius sanguineus Arnoglossus coeruleosticta Aseraggodes bahamondei Callanthias platei Caprodon longimanus Cheilopogon spilonotopterus Chironemus bicornis Chironemus delfini

Chromis meridiana Cosmocampus arctus heraldi Girella albostriata Gonorynchus greyi Gnathophis cf. smithi Gymnothorax australicola Gymnothorax bathiphylus Gymnothorax cf. obesus Gymnothorax porphyreus Hypoplectrodes semicinctum

Lotella fernandeziana

Malapterus reticulatus

Macroramphosus scolopax Maxillicosta reticulata Monocentris reedi Nemadactylus gayi Notopogon fernandezianus Odontesthes gracilis Ophidion metoecus Paralichthys fernandezianus Parapercis dockinsi

Paratrachichthys fernandezianus Paratrimma nigrimenta Polyprion oxygeneios Pseudocaranx chilensis Pseudolabrus gayi Pterygotrigla picta Scartichthys variolatus

Scolecenchelys chilensis Scorpaena fernandeziana

Scorpaena thomsoni Scorpaenodes englerti Scorpis chilensis

Seriola lalandi Sicyases brevirostris Squalus mitsukurii Suezichthys sp. Umbrina reedi
FMNH 107365-7; MNHNC P.7143, P.7149, P.7166, P.7177; UMAR 005

FMNH 109193-4; SIO 71-217.

FMNH 109195

FMNH 107273, 107275; MCZ 58150, 61199, 61202-03; UMAR 069, 071.

FMNH 107276-82; MNHNC P.7205; UMAR 027.

FMNH 107306-8; CU 51293.

FMNH 107309-14; UMAR 073, 119.

FMNH 107472; UMAR 001, 083.

FMNH 107336-39; MCZ 46175, 51018; SIO 65-657, 638,626; MNHNC P.7176; UMAR 063,098, 106.

FMNH 107340-41, 107489; MCZ 46157; SIO 65-655.

FMNH 70829, 75774, 107397-99; SIO 65-626; MNHNC P.7140, P.7186; UMAR 025

FMNH 107299; SIO 65-626.

FMNH 107331-35, 107482-86; MNHNC P.7165, P.7204; UMAR 022, 034, 116, 237.

FMNH 107244

UMAR 251.

FMNH 107251-54; SIO 65-628; MNHNC P.7154; UMAR 020.

FMNH 107255, 107467.

FMNH 107468; UMAR 125.

FMNH 107256-58, 107469-71; UMAR 035.

FMNH 107315-30; MNHNC P.7138, P.7148, P.7159, P.7179, P.7203; UMAR 012, 019, 023, 041, 052, 074, 081, 094, 102, 118,235

FMNH 107259-72; MCZ 58005; MNHNC P.7139, P.7150, P.71157, P.7172-3, P.7184, P.7190; UMAR 009, 013, 039, 049, 053, 062, 078, 090, 095, 129 .

FMNH 107422-37; MCZ 14442, 57058, 57994, 60836; MNHNC P.7146, P.7168, P.7175, P.7182, P.7193, P.7202; UMAR 018, 043, 057, 066, 080, 089, 100, 109; CU 51281 .

UMAR 252.

FMNH 107472-75, 107379; UMAR 067, 070.

FMNH 74350, 107283-90; MNHNC P.7141, P.7158, P.7163, P.7171, P.7174, P.7195; UMAR 002, 038, 046, 059, $068,130$. FMNH 107342-47, 107490; MNHNC P.7187; UMAR 026, 030, 036, 117, 122, 236.

MCZ 46675, 52384; CU 51290.

MCZ 46172, 90008; UMAR 126, 212, 223.

FMNH 107460-62; MCZ 93410; UMAR 114.

FMNH 107274, 107473-76; MCZ 44908-09; SIO 65-655; MNHNC P.6244, P.6778, P.7189; UMAR 115, 069, $071,233$.

FMNH 107441-42; UMAR 085.

FMNH 74351, 107291-98; MNHNC P.7144, P.7156; UMAR 003, 048, 091.

FMNH 107438-40.

FMNH 107481

FMNH 107300-05, 107477-78; MNHNC P.7151, P.7194; UMAR 016, 029, 037, 110, 121, 127, 234

FMNH 107401-21; MNHNC P.712, P.7180; UMAR 024, 031, 055, 065, 076, 088, 101, 105, 108; CU 51280.

UMAR 243.

FMNH 107443-59; MNHNC P.7153, P.7161, P.7169, P.7181, P.7191, P.7206; UMAR 008, 011, 014, 021, 032, 044, 054, 060, 072, 082, 093, 096, 112 .

FMNH 107245-50; MCZ 48879; MNHNC P.7152, P.7183; UMAR 061, 086, 107, 131.

FMNH 107376-77, 197380-95; MCZ 58006; SIO 65-659; MNHNC P.7137, P.7145, P.7164, P.7178, P.7192; UMAR 006 010, 017, 028, 033, 045, 051, 064, 079, 087, 099, 113, 128; CU 51286

FMNH 107378, 107396; SIO 65-637; MNHNC P. 7243; UMAR 006, 244.

FMNH 109190-92; MNHNC P.7170, P.7188, P.7196; UMAR 007, 040, 050.

FMNH 107348-58, 107360-64, 107464; MNHNC P.7142, P.7147, P.7155, P.7160, P.7167, P.7185; UMAR 004, 015, 042, 047, 077, 092, 097, 104, 111.

FMNH 107479-80; UMAR 075, 084, 120

FMNH 107463.

FMNH 107466; UMAR 232

FMNH 109197; SIO 65-629.

FMNH 107487-88. 
\& Van Leersum 2001) and, information that is required for the creation of an aquatic reserve or marine park (Castilla \& Oliva 1987).

\section{Material AND Methods}

An expedition was undertaken aboard the M/V Carlos Porter for the period of February 17- March 12, 1997. Collections were made at San Ambrosio Island from February 20-23, at San Félix from February 24-28, at Robinson Crusoe Island from February 28 to March 9, and at Alexander Selkirk Island on March 5-6. All material collected was subdivided between participating institutions: Field Museum of Natural History, Chicago (FMNH: http:// emuweb.fieldmuseum.org/fish/Query.php), Universidad del Mar (UMAR: www.ern.udelmar.cl/investigacion/ictiologia/ coleccionpeces.htm), Museo Nacional de Historia Natural, Santiago (MNHNC; Meléndez \& Cornejo 2001) and Universidad Austral de Chile, Valdivia (IZUA-P, destroyed by fire in 2008). Some specimens were studied from the Anton Bruun cruises 12 and 13 deposited at Cornell University (CU), University of Harvard's Museum of Comparative Zoology (MCZ), and Scripps Institute of Oceanography (SIO). Additional material was collected from Robinson Crusoe and Alejandro Selkirk Islands in 1999 and 2000, respectively, all specimens of which are deposited at MNHNC. Material examined by us is listed in Table 1.

The primary method for collection of fishes was scuba diving at depths of 5 to $35 \mathrm{~m}$ and the release of small (1 $\mathrm{kg}$ ) bags of powdered derris root. Some collections were taken also at intertidal pools. Other methods for collecting fishes included small mesh gill nets deployed on rocky reefs and monitored by scuba divers, small hand spears and hand nets, and collecting from the deck of the ship by hook and line, dip netting and pulling a small $10 \mathrm{ft}$ trawl down to depths of $100 \mathrm{~m}$. In addition, color photographs were taken underwater (by Marcel Duhart, Marcelo Rossi and Álvaro Sepúlveda) and in aquaria (by authors). Muscle tissue samples from most of the fishes collected, enabling interested researchers to analyze evolutionary relationships using DNA.

Taxonomy follows Eschmeyer (2010), except recent changes that have made it into the periodic updates of the online database ${ }^{1}$ and is presented in Table 2. Biogeographic accounts consider coastal fishes only as presented in Table 3.

\section{Results}

\section{Systematics}

A total of 55 species are recorded below (Table 2), of which 52 species were confirmed to inhabit the coastal waters around the Juan Fernández and Desventuradas Islands, with 41 and 43 species in each, respectively (Table 3). Juan Fernández has 5 endemic species (12.2\%; Sicyases brevirostris, Odontesthes gracilis, Chironemus delfini, Parapercis dockinsi, and Paratrimma nigrimenta); three new records for these islands are Gymnothorax cf. obesus, Gnathophis sp. and Suezichthys sp.; Desventuradas Islands have only 2 endemics (4.6\%; Chromis meridiana and Paratrimma urospila) and 4 new records: Scorpaenodes englerti, Maxillicosta reticulata, Suezichthys sp., and Aseraggodes bahamondei.

The following account of species includes only those collected during the IOC-97 expedition, plus additional species collected since then by one of the authors (BD) and for which specimens exist in collections and their identifications verified. The following species have not been included in the list below or Table 2: Mendosoma fernandezianus (Latridae) is considered nomin dubium by Gon \& Heemstra (1987); Paralabrax humeralis, Micropogon fasciatus, Chromis crusma, Trachurus murphyi, and Paralichthys adspersus are incidental species on the islands from the continental coast; Scolecenchelys profundorum (IZUA 1525) and Physiculus longicavis (IZUA 1678) are deep water species from the Nazca Ridge collected at Desventuradas Islands only; Trachypoma macracanthus (IZUA 1711, Rojas \& Pequeño 1996, 1998a,c) was not collected by us; and Pseudocaranx dentex is a misidentification of $P$. chilensis. The authors were unable to corroborate Pentaceros capensis, Synodus capricornensis, and Kyphosus cinerascens (all cited as present in Desventuradas Islands by Pequeño \& Lamilla 2000) as collected at any of these islands and they are not considered in the systematic part of this study.

SQUALIDAE

Dogfishes / Tollos

\footnotetext{
${ }^{1}$ http://research.calacademy.org/ichthyology/catalog/fishcatmain.asp
} 
Table 2. Systematic list of coastal fishes from Juan Fernández and Desventuradas Islands / Lista sistemática de peces costeros de las islas de Juan Fernández y Desventuradas

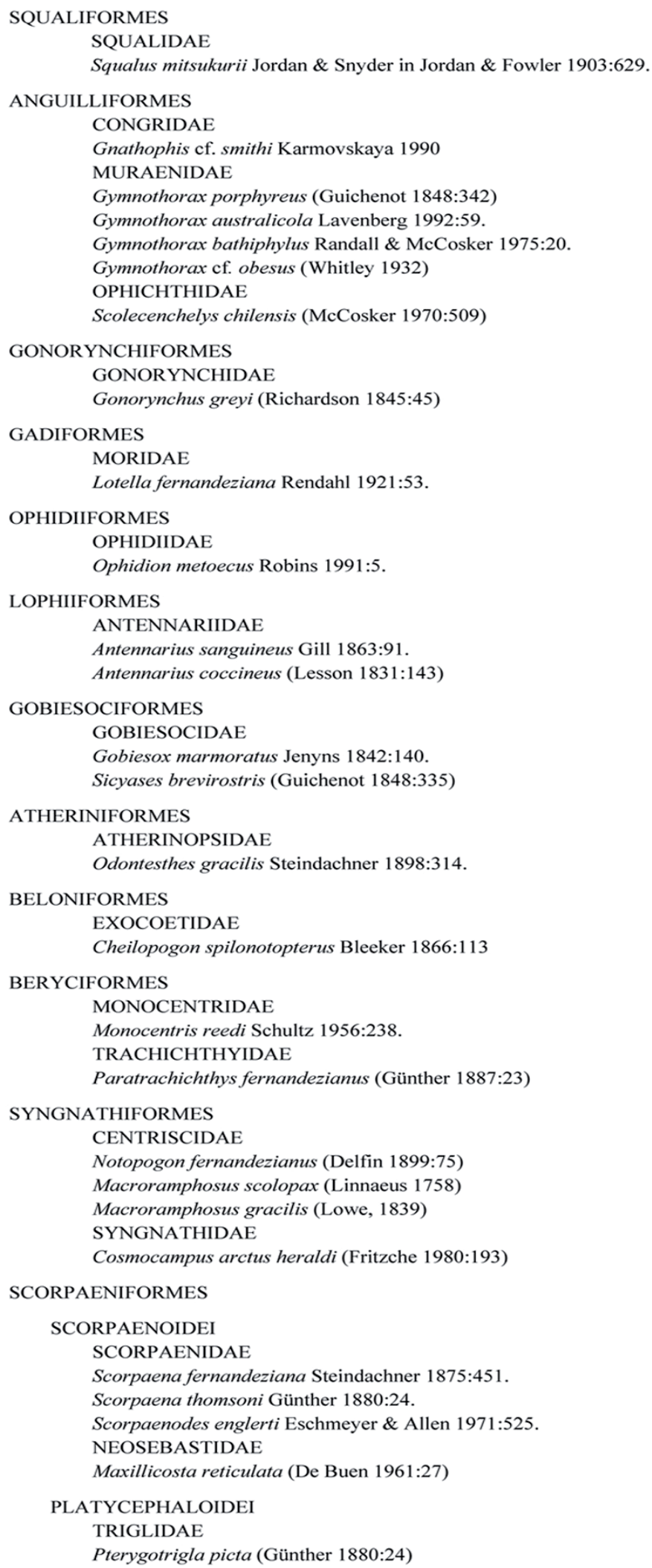


Continuación de Tabla 2 / Continuation of Table 2

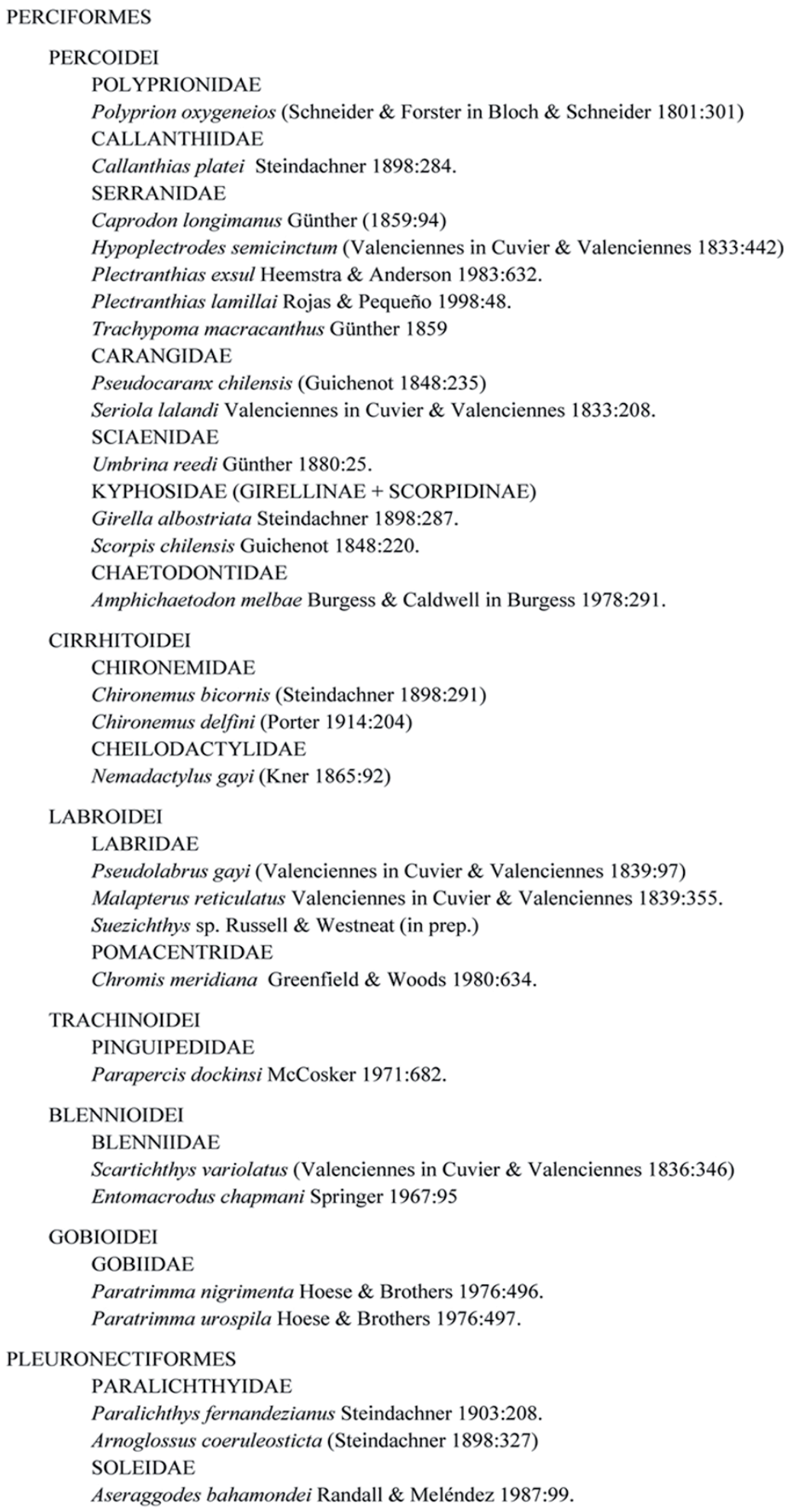


Squalus mitsukurii Jordan \& Snyder in Jordan \& Fowler, 1903

Squalus mitsukurii Jordan \& Snyder in Jordan \& Fowler 1903:629. Type locality: Misaki, Japan.

This species is quite abundant in Juan Fernández, at least in austral summer, as evidenced by gill-net catches (Arana 2000). Female specimens collected at San Ambrosio Island all contained many embryos, indicating they probably reproduce at these islands as reported for Juan Fernández by Pequeño \& Toledo (1994).

\section{CONGRIDAE}

Conger eels / anguilas

Gnathophis ef. smithi Karmovskaya, 1990. Fig. 3

Gnathophis smithi Karmovskaya 1990: 767 Type

Locality: E. Pacific, $25^{\circ} 43^{\prime}$ S, $85^{\circ} 22^{\prime} \mathrm{W}, 200-185$ m.

Gnathophis parini Karmovskaya, 1990

Gnathophis parini Karmovskaya 1990: 764 Type

Locality: E. Pacific, $25^{\circ} 34^{\prime} \mathrm{S}, 89^{\circ} 09^{\prime} \mathrm{W}, 540-560 \mathrm{~m}$.

This species was described based on specimens collected at the juncture of the Nazca and Salas \& Gómez ridges at depths over $100 \mathrm{~m}$. A specimen of $371 \mathrm{~mm}$ TL was collected $20 \mathrm{~m}$ deep by hand during a night dive in Robinson Crusoe Island (UMAR 251) and another of 800 $\mathrm{mm}$ TL was collected at dusk at $50 \mathrm{~m}$ by hook and line. It is attributed to this species based on the black margin of the median dorsal fins and 170 anal fin rays, however, its anterior lateral line pores are ventral (except for the second pore) as in G. parini. The other specimen of Gnathophis collected at Robinson Crusoe Island was identified as $G$. catalinensis (SIO65-658: $371 \mathrm{~mm} \mathrm{TL}$ ). Species of this genus are nocturnal and appear to be resistant to rotenone or hide further when it is applied, which may explain why so few specimens have been collected. This will be treated as one species for the biogeographic analysis.

\section{MURAENIDAE}

Moray eels / Morenas

Gymnothorax porphyreus (Guichenot, 1848). Fig. 3.

Murenophis porphyreus Guichenot in Gay 1848:342. Type locality: Chile

?Muraena chilensis Günther, 1871. Type Locality: Chile.

Gymnothorax wieneri Sauvage, 1883. Type Locality: Chile, Perú.
Gymnothorax obscurirostris Rendahl, 1921. Type Locality: Easter Island.

This species has been described in detail by Randall \& McCosker (1975:23) for Easter Island and by Meléndez \& Villalba (1992) for the Juan Fernández Islands. It is certainly the most common eel in both island groups surveyed by us and ubiquitous in its aggressive display behavior. Its coloration is mostly dark brown irregular blotches over a pale yellow background. Distributional range is from Lord Howe and Norfolk islands in the West Pacific (Francis \& Randall 1993) to continental Perú and Chile in the East.

Gymnothorax australicola Lavenberg, 1992. Fig. 3.

Gymnothorax australicola Lavenberg 1992:59. Type Locality: Easter Island, Anakena Cove.

This species was described for the light brown, plaincolored morays with a black eye ring, white blotches around the last two infraorbital pores and last three mandibular pores, and a single branchial pore. Known from Lord Howe Island (Francis 1993), Rapa, Ducie Atoll (Pitcairn Islands), Easter Island, San Félix and San Ambrosio Islands (FMNH 107251-107254, MNHNC P7154).

Gymnothorax bathiphylus Randall \& McCosker, 1975.

Gymnothorax bathiphylus Randall \& McCosker 1975:20. Type Locality: Easter Island, off Mt. Tere Vaka.

Collection of two more specimens in Desventuradas (FMNH 107255, 107467) complements this species range extension to the East presented by Pequeño \& Lamilla (1996a). Described in detail by Randall \& McCosker (1975:20), it is distinguished from the other morays on Desventuradas by its light yellowish-gray background with irregularly-placed roundish dark blotches. Visible, small black dots of lateral line pores around and behind head. Found in Easter Island and Desventuradas Islands.

Gymnothorax cf. obesus (Whitley, 1932). Fig. 3.

Uropterygius obesus Whitley 1932:329. Type locality: off Montague Island, NSW, Australia.

Gymnothorax griffini Whitley \& Phillipps 1939:229. Type locality: White Island, Bay of Plenty, New Zealand.

Two large specimens collected in lobster traps 30-40 m deep at Robinson Crusoe Island (FMNH 107468) and Santa Clara Island (UMAR 125). They are similar in shape, 


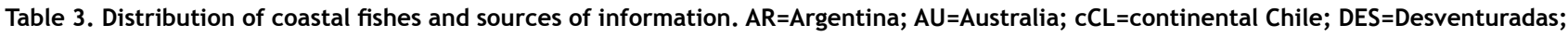
GI= Galápagos Is.; JF= Juan Fernández; KI=Kermadec Is.; LH=Lord Howe; NI=Norfolk Is.; NZ=New Zealand; PE=Perú; PI=Pitcairn Is.; SA= South America; sAU= southern Australia; S\&G=Salas y Gómez Is.; UR=Uruguay / Distribución de peces costeros y fuentes de información. AR=Argentina; $\mathrm{AU}=$ Australia; $\mathrm{CCL}=$ Chile continental; $\mathrm{DES}=$ Desventuradas; $\mathrm{Gl}=\mathrm{Is}$. Galápagos; JF=Juan Fernández; $\mathrm{Kl}=\mathrm{Is}$. Kermadec; $\mathrm{LH}=\mathrm{Lord} \mathrm{Howe}$; $\mathrm{Nl}=\mathrm{Is}$. $\mathrm{Norfolk}$; $\mathrm{NZ}=$ Nueva Zelandia; $\mathrm{PE}=$ Perú; $\mathrm{Pl}=\mathrm{Is}$. Pitcairn; $\mathrm{SA}=$ Sud América; $\mathrm{SAU}=$ sur de Australia; $\mathrm{S} \& \mathrm{G}=\mathrm{Is}$. Salas y Gómez; UR=Uruguay

\begin{tabular}{|c|c|c|c|c|c|c|}
\hline Taxa & SA & JF & DES & $\begin{array}{l}\text { Easter } \\
\text { Island }\end{array}$ & $\begin{array}{l}\text { Western } \\
\text { Pacific }\end{array}$ & Authors \\
\hline \multicolumn{7}{|l|}{ ANGUILLIFORMES } \\
\hline Gymnothorax porphyreus & $\begin{array}{l}\text { PE, } \\
\text { cCL }\end{array}$ & $\mathrm{X}$ & $\mathrm{X}$ & $\mathrm{X}$ & LH, NI & $\begin{array}{l}\text { Randall \& McCosker 1975; } \\
\text { Francis } 1993\end{array}$ \\
\hline Gymnothorax australicola & & & $\mathrm{X}$ & $\mathrm{X}$ & $\begin{array}{l}\text { Rapa, LH, } \\
\text { PI, Ducie }\end{array}$ & Lavenberg 1992 \\
\hline Gymnothorax bathiphylus & & & $\mathrm{X}$ & $\mathrm{x}$ & & Randall \& McCosker 1975 \\
\hline Gymnothorax cf. obesus & & $\mathrm{X}$ & & & $\begin{array}{l}\mathrm{AU}, \mathrm{NZ} \\
\mathrm{NI}\end{array}$ & Gomon et al. 1994 \\
\hline Gnathophis cf. smithi & & $\mathrm{X}$ & $\mathrm{X}$ & Nazca ridge & $\mathrm{AU}, \mathrm{NZ}$ & Karmovskaya \& Paxton 2000 \\
\hline Scolecenchelys chilensis & & $\mathrm{x}$ & $\mathrm{X}$ & & & Castle \& McCosker 1999 \\
\hline \multicolumn{7}{|l|}{ GONORYNCHIFORMES } \\
\hline Gonorynchus greyi & & & $\mathrm{X}$ & $\mathrm{X}$ & $\begin{array}{l}\mathrm{AU}, \mathrm{NZ} \\
\mathrm{LH}, \mathrm{NI}\end{array}$ & Grande 1999 \\
\hline \multicolumn{7}{|l|}{ GADIFORMES } \\
\hline Lotella fernandeziana & & $\mathrm{X}$ & $\mathrm{X}$ & & & \\
\hline \multicolumn{7}{|l|}{ OPHIDIIFORMES } \\
\hline Ophidion metoecus & & $\mathrm{X}$ & $\mathrm{X}$ & & & Robins 1991 \\
\hline \multicolumn{7}{|l|}{ LOPHIIFORMES } \\
\hline Antennarius sanguineus & & & $\mathrm{X}$ & $\mathrm{X}$ & $\mathrm{X}$ & Pietsch \& Grobecker 1987; \\
\hline Antennarius coccineus & $\mathrm{X}$ & & $\mathrm{X}$ & $\mathrm{X}$ & $\mathrm{AU}, \mathrm{LH}$ & Francis 1993 \\
\hline \multicolumn{7}{|l|}{ GOBIESOCIFORMES } \\
\hline Gobiesox marmoratus & $\mathrm{X}$ & $\mathrm{X}$ & & & & \\
\hline Sicyases brevirostris & & $\mathrm{X}$ & & & & Briggs 1955 \\
\hline \multicolumn{7}{|l|}{ ATHERINIFORMES } \\
\hline Odontesthes gracilis & & $\mathrm{X}$ & & & & Dyer \& Gosztonyi 1999 \\
\hline \multicolumn{7}{|l|}{ BELONIFORMES } \\
\hline Cheilopogon spilonotopterus & GI & $\mathrm{X}$ & $\mathrm{X}$ & $\mathrm{X}$ & $\mathrm{X}$ & Parin 1999 \\
\hline \multicolumn{7}{|l|}{ BERYCIFORMES } \\
\hline Monocentris reedi & & $\mathrm{X}$ & $X$ & Nazca ridge & & Kotlyar 1985 \\
\hline Paratrachichthys fernandezianus & & $\mathrm{X}$ & $\mathrm{X}$ & & & \\
\hline \multicolumn{7}{|l|}{ SYNGNATHIFORMES } \\
\hline Notopogon fernandezianus & $\begin{array}{l}\text { AR,UR } \\
\text { cCL }\end{array}$ & $\mathrm{X}$ & & S\&G & & Duhamel 1995 \\
\hline Macroramphosus scolopax & & $\mathrm{X}$ & $\mathrm{X}$ & $\mathrm{X}$ & $\mathrm{AU}, \mathrm{NZ}$ & Pequeño \& Lamilla 2000 \\
\hline Macroramphosus gracilis & & & $\mathrm{X}$ & $\mathrm{X}$ & & \\
\hline Cosmocampus arctus heraldi & & $\mathrm{X}$ & $\mathrm{X}$ & & & Dawson 1985 \\
\hline \multicolumn{7}{|l|}{ SCORPAENIFORMES } \\
\hline Scorpaena fernandeziana & & $\mathrm{X}$ & $\mathrm{X}$ & & & Meléndez \& Villalba 1992 \\
\hline Scorpaena thomsoni & & $\mathrm{X}$ & $\mathrm{X}$ & & & \\
\hline Scorpaenodes englerti & & & $\mathrm{X}$ & $\mathrm{X}$ & & Eschmeyer \& Allen 1971 \\
\hline Maxillicosta reticulata & & $\mathrm{X}$ & $\mathrm{X}$ & & & Eschmeyer \& Poss 1976 \\
\hline Pterygotrigla picta & & $\mathrm{X}$ & & $\begin{array}{c}\mathrm{X} \\
\text { S\&G } \\
\text { Nazca ridge }\end{array}$ & sAU, NZ & $\begin{array}{l}\text { Randall et al. 2005; Golovan \& } \\
\text { Pakhorukov 1987; Kuiter } 1993\end{array}$ \\
\hline
\end{tabular}


Continuación de Tabla 3 / Continuation of Table 3

\begin{tabular}{|c|c|c|c|c|c|c|}
\hline Taxa & SA & $\mathrm{JF}$ & DES & $\begin{array}{l}\text { Easter } \\
\text { Island }\end{array}$ & $\begin{array}{l}\text { Western } \\
\text { Pacific }\end{array}$ & Authors \\
\hline \multicolumn{7}{|l|}{ PERCIFORMES } \\
\hline Caprodon longimanus & & $\mathrm{X}$ & $\mathrm{X}$ & $\mathrm{X}$ & $\mathrm{X}$ & Pequeño \& Lamilla 1996b \\
\hline Hypoplectrodes semicinctum & & $\mathrm{X}$ & $\mathrm{X}$ & $\mathrm{X}$ & & Randall et al. 2005 \\
\hline Plectranthias exsul & & $\mathrm{X}$ & $\mathrm{X}$ & & & Anderson \& Baldwin 2000 \\
\hline Trachypoma macracanthus & & & $\mathrm{X}$ & $\mathrm{X}$ & $\mathrm{X}$ & Rojas \& Pequeño 1998 \\
\hline Pseudocaranx chilensis & & $\mathrm{X}$ & $\mathrm{X}$ & & & \\
\hline Seriola lalandi & $\mathrm{X}$ & $\mathrm{X}$ & $\mathrm{X}$ & $\mathrm{X}$ & $\mathrm{X}$ & Grove \& Lavenberg 1997 \\
\hline Umbrina reedi & & $\mathrm{X}$ & $\mathrm{X}$ & & & Walker \& Radford 1992 \\
\hline Girella albostriata & & $\mathrm{X}$ & $\mathrm{X}$ & & & \\
\hline Scorpis chilensis & & $\mathrm{X}$ & $\mathrm{X}$ & & & \\
\hline Amphichaetodon melbae & & $\mathrm{X}$ & $\mathrm{X}$ & $\mathrm{X}$ & & $\begin{array}{l}\text { Di Salvo et al. 1988; Meléndez \& } \\
\text { Villalba } 1992\end{array}$ \\
\hline Chironemus bicornis & & $\mathrm{X}$ & $\mathrm{X}$ & & & Meléndez \& Dyer 2010; \\
\hline Chironemus delfini & & $\mathrm{X}$ & & & & Burridge et al. 2006 \\
\hline Nemadactylus gayi & & $\mathrm{X}$ & $\mathrm{X}$ & & & Burridge 1999 \\
\hline Pseudolabrus gayi & & $\mathrm{X}$ & $\mathrm{X}$ & & & \\
\hline Malapterus reticulatus & & $\mathrm{X}$ & $\mathrm{X}$ & & & Russell 1988 \\
\hline Suezichthys sp. & & $\mathrm{X}$ & $\mathrm{X}$ & & & Russell \& Westneat in prep. \\
\hline Chromis meridiana & & & $\mathrm{X}$ & & & Greenfield \& Woods 1980 \\
\hline Parapercis dockinsi & & $\mathrm{X}$ & & & & \\
\hline Scartichthys variolatus & & $\mathrm{X}$ & $\mathrm{X}$ & & & Pequeño \& Sáez 2004 \\
\hline Entomacrodus chapmani & & & $\mathrm{X}$ & $\mathrm{X}$ & & \\
\hline Paratrimma nigrimenta & & $\mathrm{X}$ & & & & Hoese \& Brothers 1976 \\
\hline Paratrimma urospila & & & $\mathrm{X}$ & & & \\
\hline \multicolumn{7}{|l|}{ PLEURONECTIFORMES } \\
\hline Paralichthys fernandezianus & & $\mathrm{X}$ & $\mathrm{X}$ & & & \\
\hline Arnoglossus coeruleosticta & & $\mathrm{X}$ & $\mathrm{X}$ & & & \\
\hline Aseraggodes bahamondei & & & $\mathrm{X}$ & $\mathrm{X}$ & LH, NI, KI & Francis 1993; Francis \& Randall 1993 \\
\hline $\mathrm{N}^{\circ}$ coastal species & & 41 & 43 & & & \\
\hline $\mathrm{N}^{0}$ endemic species & & 5 & 2 & & & \\
\hline TOTAL $\mathrm{N}^{\mathrm{o}}$ endemic species & \multicolumn{6}{|c|}{22} \\
\hline TOTAL No shared species & \multicolumn{6}{|c|}{32} \\
\hline TOTAL N ${ }^{\circ}$ coastal species & \multicolumn{6}{|c|}{52} \\
\hline
\end{tabular}


size and pattern of speckling to G. obesus from Southern Australia, northern New Zealand and Kermadec Islands (Gomon et al. 1994:207). Teeth in jaw roughly 25 in single series, anterior enlarged fang-like and depressible; vomerine teeth small. Mouth large (44\% head length) does not fully close, reaching well beyond eyes. Lower jaw slightly prognathous, upper jaw blunt. Eyes small, roughly the same size as branchial opening, and continuous with surrounding skin. Branchial opening slanted posteriorly, below midline. Two branchial pores, three mandibular pores, four suborbital pores, and posterior nasal opening above and in front of eye. Head length roughly 7 times total length; preanal length about equal to tail length, posterior third laterally compressed. No specimens found recorded from Desventuradas Islands.

\section{OPHICHTHIDAE}

Worm eels / Anguila fideo

Scolecenchelys chilensis (McCosker, 1970). Fig. 3.

Muraenichthys chilensis McCosker 1970:509. Type locality: Robinson Crusoe Island, South of Pta. Suroeste. $33^{\circ} 37^{\prime} 15^{\prime \prime} \mathrm{S}, 78^{\circ} 55^{\prime} 05^{\prime \prime} \mathrm{W}$.

Easily distinguished from other eels of the islands in being long, slender and living in the sand and debris between rocks and crevices. Body of a uniformly tanned to light brown color. Snout long, upper jaw prognathous, and lower jaw reaching the anterior nostrils. Both nostrils are along side of upper jaw, projecting ventrally. Only recently Castle $\&$ McCosker (1999) recognized this group of myrophine worm eels as a genus distinct from Muraenichthys, though no phylogenetic hypothesis was provided. Found in both Juan Fernández and Desventuradas Islands.

\section{GONORYNCHIDAE}

Sandfish

Gonorynchus greyi (Richardson, 1845). Fig. 3.

Rynchana greyi Richardson 1845:45. Type locality: W. Australia and Port Nicholson, Cook's Strait, New Zealand.

Gonorynchus parvimanus Ogilby 1911:34. Type locality: Moreton Bay, Queensland, Australia.

Predominantly found in waters off the east and west coasts of Australia (Grande 1999), this species was trawled at a depth of $100 \mathrm{~m}$ over sandy bottom between the St. Peterborough Cathedral Rock and San Félix Island (FMNH 107244), presenting another example of extreme eastern range extension for an Indo-Pacific or Western Pacific group.

MORIDAE

Morid cods / Congrios

Lotella fernandeziana Rendahl, 1921. Fig. 3.

Lotella fernandeziana Rendahl 1921:53. Type locality: Masatierra (Robinson Crusoe), Juan Fernández Islands.

Abundant in most rotenone collections made on both island groups, recently recorded at Desventuradas (Meléndez \& Pequeño 1996) but not recorded in Nazca Ridge (Parin 1991) or Easter Island collections (Di Salvo et al. 1988). This genus, of only five species, is mostly western Pacific in distribution, except for this species and L. schuetta found in eastern Indian Ocean, Western Australia. Found in caves and under rocks together with Paratrachichthys fernandezianus.

\section{OPHIDIIDAE}

Cusk eels / Congrios, Brótulas

Ophidion metoecus Robins, 1991. Fig. 3.

Ophidion metoecus Robins 1991:5. Type locality: Robinson Crusoe Island, Juan Fernández Islands.

Uncommon in collections. Though no live color information was available at the time of description, Robins (1991) was correct in his assumption that coloration was similar to $O$. exul, however the lateral line is not whitish as he suggested. Ophidion exul remains to be the larger species; the longest specimen of $O$. metoecus collected by us was $113 \mathrm{~mm} \mathrm{SL}$ (FMNH 107460-107462).

\section{ANTENNARIIDAE}

Frogfishes / Pez Pescador

Antennarius sanguineus Gill, 1863.

Antennarius sanguineus Gill 1863:91. Type locality: Cape San Lucas, Baja California, Mexico.

Antennarius leopardinus Günther 1864:151. Type locality: Pacific coast of Panamá.

Antennarius tagus Heller \& Snodgrass 1903:226. Type locality: Tagus Cove, Isabela I. [Albemarle I.], Galápagos Islands.

Antennarius coccineus (Lesson, 1831). Fig. 3. 

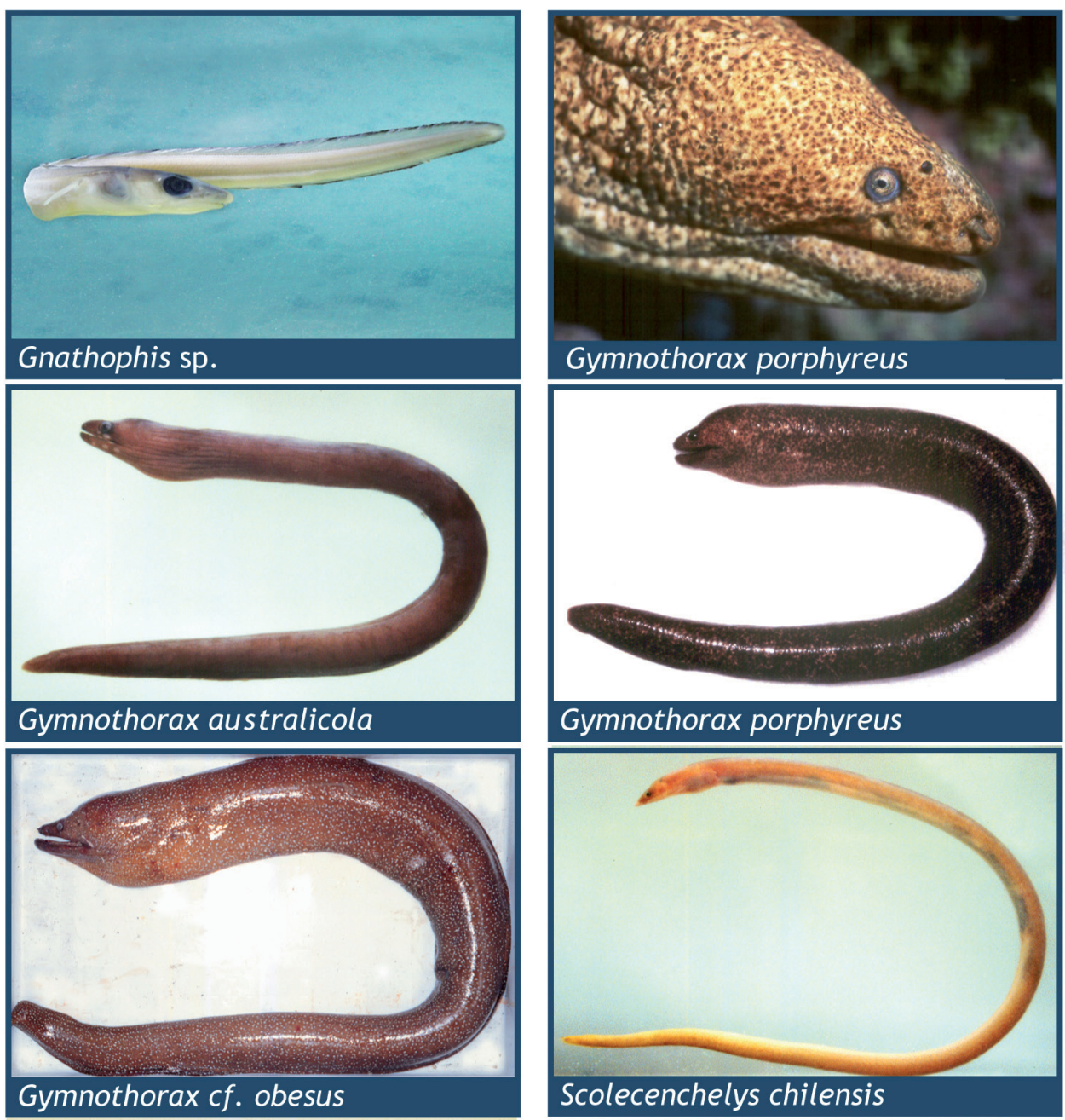

Gymnothorax porphyreus
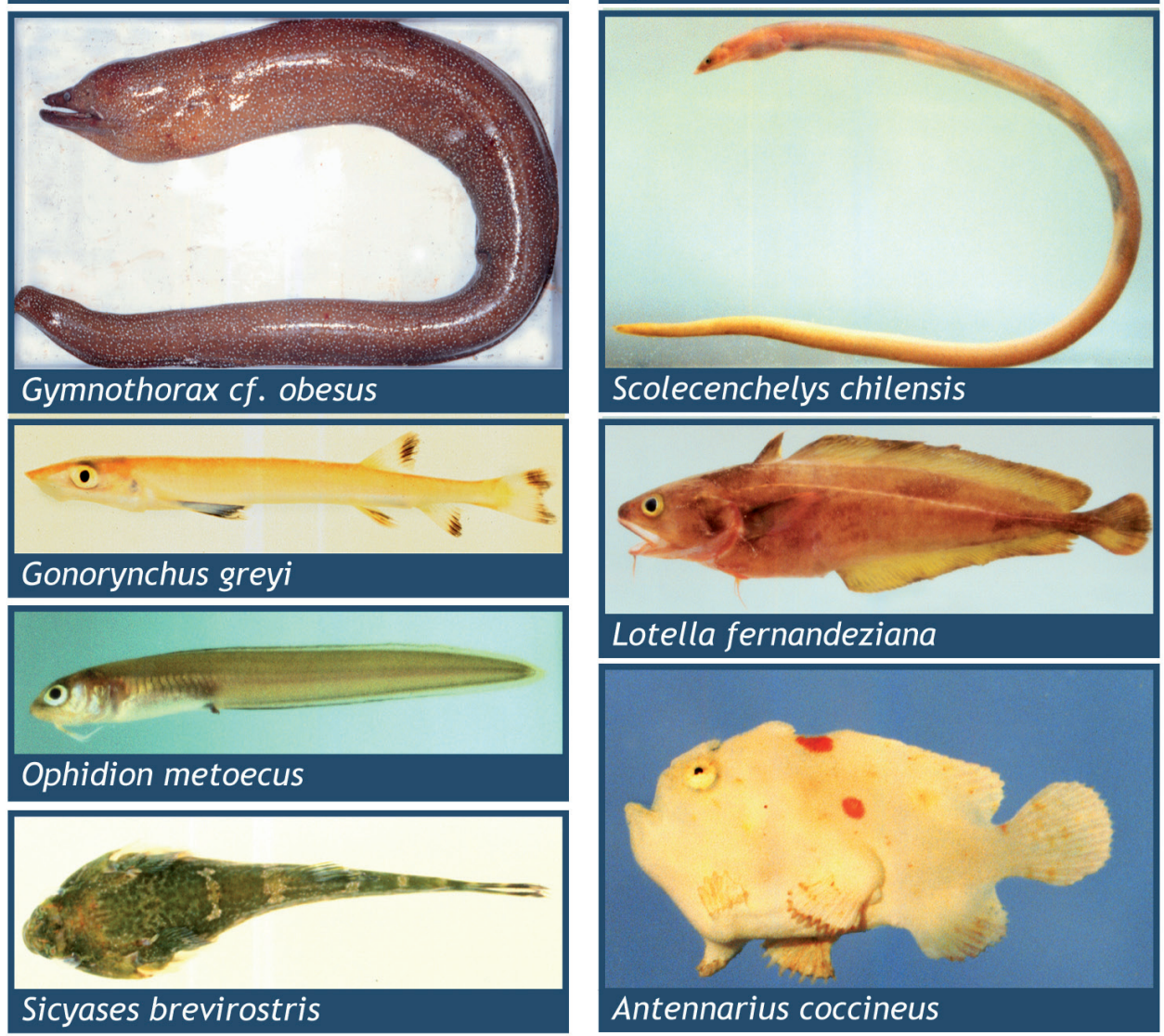

Figure 3. Species of the fish families Congridae, Muraenidae, Ophichthidae, Gonorynchidae, Moridae, Ophidiidae, Antennariidae, and Gobiesocidae. Underwater photo of G. porphyreus by A. Sepúlveda / Especies de peces de las familias Congridae, Muraenidae, Ophichthidae, Gonorynchidae, Moridae, Ophidiidae, Antennariidae y Gobiesocidae. Foto submarina de G. porphyreus por A. Sepúlveda 
Chironectes coccineus Lesson (ex Cuvier) 1831:143. Type locality: Mauritius.

Antennarius drombus Jordan \& Evermann 1903:207. Type locality: Waikiki near Honolulu, Oahu I., Hawaiian Islands.

Antennarius nexilis Snyder 1904:537. Type locality: Honolulu, Oahu I., Hawaiian Islands.

Antennarius stigmaticus Ogilby 1912:63. Type locality: Moreton Bay, Queensland, Australia.

Antennarius leucus Fowler 1934:512. Type locality: Durban, Natal, South Africa.

Abantennarius neocaledoniensis Le Danois 1964:126. Type locality: New Caledonia.

Antennarius moai Allen 1970:521. Large tide pool between Hanga Roa and Hanga Piko, Easter Island.

Antennarius immaculatus Le Danois 1970:91. Type locality: Eilat, Gulf of Aqaba.

Both species were collected at Desventuradas Islands (FMNH 109193-95) complementing a previous collection at San Félix by Anton Bruun cruises. As commented by Pietsch \& Grobecker (1987:160), both these species are morphologically similar, occur sympatrically in the southeastern Pacific, and are easily distinguishable from each other only when specimens are over $25 \mathrm{~mm}$ SL. Antennarius sanguineus is distinguished in that it has darkly pigmented swellings of the esca and dark rounded spots on the belly, both features lacking in A. coccineus. When specimens are under $25 \mathrm{~mm} \mathrm{SL}$, only the difference in dorsal fin ray counts separates $A$. sanguineus (13 rays) from $A$. coccineus (12 rays).

\section{GOBIESOCIDAE}

Clingfishes / Pejesapos

Gobiesox marmoratus Jenyns, 1842.

Gobiesox marmoratus Jenyns 1842:140. Type locality: Off Lemuy Island, Chiloé Archipelago, Chile

Gobiesox nigricans Philippi in Delfin 1899:122. Type locality: Chonos Island, Chile.

Sicyases brevirostris (Guichenot, 1848). Fig. 3.

Gobiesox brevirostris Guichenot 1848:335. Type locality: Juan Fernández Islands.

Sicyases hildebrandi Schultz 1944:61. Type locality: Cumberland Bay, Juan Fernández Islands.
Both species occur at Robinson Crusoe and A. Selkirk Islands, but are apparently not syntopic as both were not collected at the same time and location. Vera \& Pequeño (2001) compared the insular populations of Sicyases with those of continental Chile and supported the differentiation between insular and continental species. Vera \& Pequeño (2001) followed De Buen (1960) in considering the insular species as $S$. hildebrandi rather than the earlier $S$. brevirostris. Sicyases brevirostris is considered by us as senior synonym of S. hildebrandi following Briggs' (1955) conclusion based on examination of type material, rather than following De Buen's doubt that Guichenot (1848) ever had specimens from the islands and his ascription of $S$. brevirostris as junior synonym of $S$. sanguineus. Briggs (1955) distinguished Sicyases brevirostris from $S$. sanguineus by a smaller head ( $>30 \%$ vs. $\leq 30 \% \mathrm{SL})$ and more anal fin rays (9 vs. 7-8). Vera \& Pequeño's (2001) study included an additional feature to distinguish both species: S. brevirostris with smooth skin and S. sanguineus with rugose skin except in the smallest specimens. Further research is needed, possibly at the molecular level, in order to determine whether these island populations are indeed distinct from their continental counterparts and if there has been any dispersal. Neither species is present at Desventuradas Islands.

\section{ATHERINOPSIDAE}

Silverside / Pejerrey

Odontesthes gracilis (Steindachner, 1898). Fig. 4.

Chirostoma gracile Steindachner 1898:314. Type locality: Cumberland Bay, Robinson Crusoe Island, Juan Fernández Islands.

Found in schools in caves and inlets very close to the surface or under the pier at Cumberland Bay (UMAR 126, 212, 223). Semi transparent when alive with greenish hues above the silvery lateral band. Presence of this species at Alejandro Selkirk Island was confirmed in Dyer (2000), but no specimens were collected at Desventuradas.

\section{EXOCOETIDAE}

Flyingfishes / Pez Volador

\section{Cheilopogon spilonotopterus (Bleeker, 1866).}

Exocoetus spilonotopterus Bleeker 1866:113. Type locality: Padang, Sumatra, Indonesia.

Pectoral and pelvic fins extended to anal fin or beyond. Pectoral fin uniformly brown with transparent margin. Lower jaw equal or slightly prognathous. Black blotch on dorsal fin. Anal-fin origin behind vertical that passes 
by third dorsal-fin ray. Dorsal part of body black or dark blueish (FMNH 107472).

\section{MONOCENTRIDAE}

Pinecone fishes

Monocentris reedi Schultz, 1956. Fig. 4.

Monocentris reedi Schultz 1956:238. Type locality: Off Robinson Crusoe Island, Juan Fernández Islands.

This species is the sole representative of the family in the southeastern Pacific, with the other three congeneric species in Japan, Australia and New Zealand waters. This species was collected at Desventuradas Islands only, in intertidal pools, in caves at 10-30 m, and trawled at 50-100 m (FMNH 107283-107290), however also found at depths up to $250 \mathrm{~m}$ (Kotlyar 1985). All specimens encountered in museum collections are from Desventuradas or Nazca Ridge (Kotlyar 1985, 1996), except the type specimen (USNM 164227) said to have been from Juan Fernández. De Buen (1959a) described another specimen from Selkirk Island though no specimen is available and mentioned as extremely rare by fishermen there. Dorsal and pelvic spines robust with a locking mechanism similar to sticklebacks and some catfishes.

\section{TRACHICHTHYIDAE}

Roughies, Sandpaper fishes / Pez Soldado

Paratrachichthys fernandezianus (Günther, 1887) Fig. 4.

Trachichthys fernandezianus Günther 1887:23. Type locality: Juan Fernández Islands.

A very abundant species, aggregated in caves together with Lotella fernandeziana and less often with Monocentris reedi, and found in Desventuradas and Juan Fernández islands (FMNH 107291-107298). Live color is coppery brown, turning to brown and gray in formaline. Single representative of the genus in the southeastern Pacific, with nine other congeners found worldwide.

\section{CENTRISCIDAE}

Bellowsfish / Pez picaflor

Notopogon fernandezianus (Delfin, 1899).

Centriscus fernandezianus, Delfin 1899:75. Type locality: Juan Fernández Islands.

Macrorhamphosus schoteli Weber 1909: 76,77. Type locality: Western Atlantic between Bahia and Montevideo, Uruguay.
Duhamel (1995) revised the genus and concluded this species is restricted to the southeastern Pacific (Juan Fernández to Nazca and Salas \& Gómez ridges) and southwestern Atlantic (southern Brazil, Uruguay, and northern Argentina). Meléndez \& Villalba (1992) described and photographed a specimen collected from Robinson Crusoe (MNHNC P6761). Specimens were also collected by R/V Anton Bruun (SIO65-648, 653, 656). Though no specimen has been reported from Desventuradas Islands it is presumed to be present there as well.

Longspine snipefish / Pez Picaflor

Macroramphosus scolopax (Linnaeus, 1758)

Balistes scolopax Linnaeus 1758: 329 Type Locality: Mediterranean Sea.

Centriscus velitaris Pallas 1770 Type Locality: Ambon Island, Moluccas Islands, Indonesia.

Silurus cornutus Forsskal 1775. Type Locality: Marseille, France.

Macroramphosus gracilis (Lowe, 1839)

Centriscus gracilis, Lowe 1839: 86. Type Locality: Off Madeira.

Macroramphosus hawaiiensis Gilbert 1905. Type Locality: Near Laysan Island, Leeward Island, Hawaiian Islands, U.S.A., Albatross Sta. 3940, 59-70 fm.

Macroramphosus molleri Whitley 1930. Type Locality: Maroubra Beach near Sydney, NSW Australia.

Two specimens of Macroramphosus were collected at Robinson Crusoe Island at a depth of $20 \mathrm{~m}$ during a night dive (UMAR 252), however, specimens of both species have been trawled at greater depths of 140-155 m (SIO65647,649,656) at Robinson Crusoe Island, at San Félix Island (De Buen 1963), and observed underwater at 260 $m$ on the Nazca and Salas \& Gómez submarine ranges (Golovan \& Pakhorukov 1987). Both species seem to inhabit sympatrically all oceans of the world (Matthiessen et al. 2003).

\section{SYNGNATHIDAE}

Pipefishes / Pez Aguja

Cosmocampus arctus heraldi (Fritzche, 1980). Fig. 4.

Bryx (Simocampus) heraldi Fritzsche 1980:193. Type locality: San Félix Island.

A single male specimen was collected at San Ambrosio Island, with numerous larvae in pouch (FMNH 107299). 

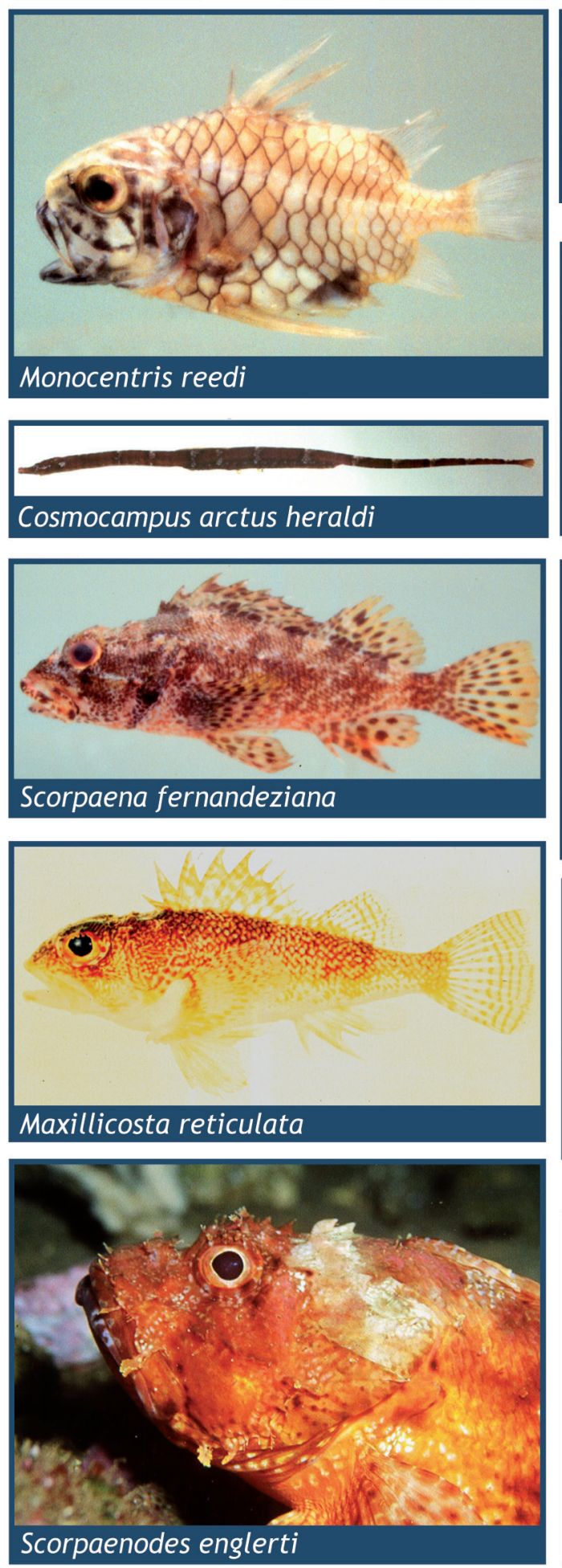
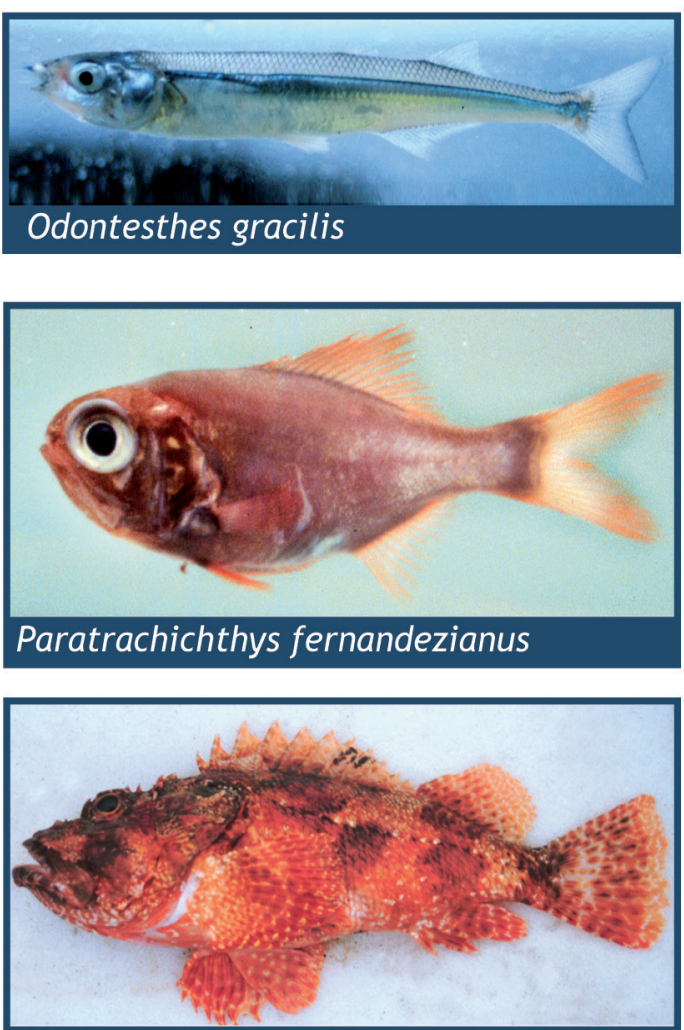

\section{Scorpaena thomsoni}
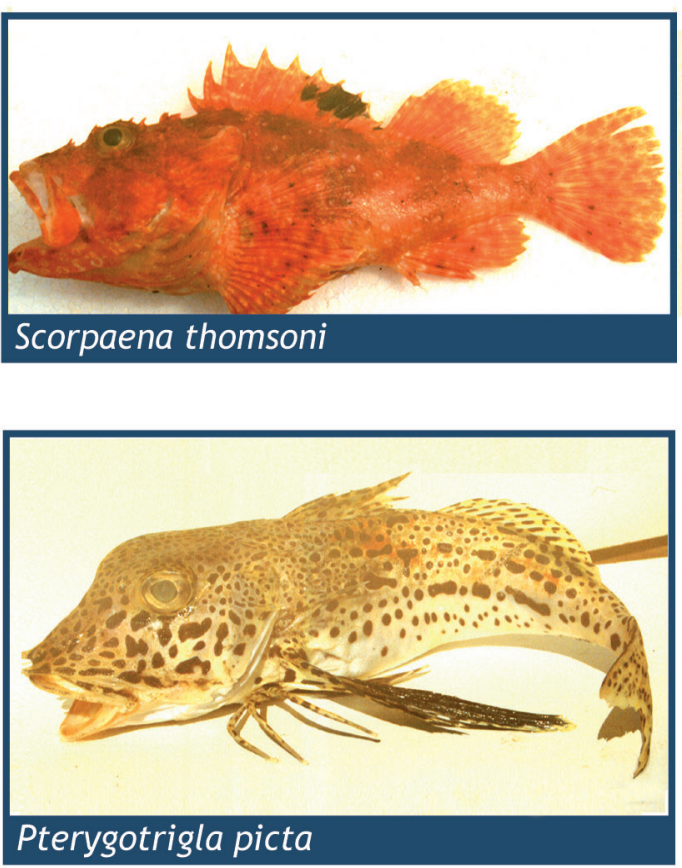

Figure 4. Species of the fish families Atherinopsidae, Monocentridae, Trachichthyidae, Syngnathidae, Scorpaenidae, Neosebastidae, and Triglidae. Underwater photo of S. englerti by A. Sepúlveda / Especies de peces de las familias Atherinopsidae, Monocentridae, Trachichthyidae, Syngnathidae, Scorpaenidae, Neosebastidae y Triglidae. Foto submarina de S. englerti por A. Sepúlveda 
Distinguished from other subspecies of $C$. arctus in having a dorsal-fin base longer than head length (Dawson 1985). Present in both island groups.

\section{SCORPAENIDAE}

Scorpionfishes / Cabrillas, Escorpénidos

Scorpaena fernandeziana Steindachner 1875. Fig. 4.

Scorpaena fernandeziana Steindachner 1875:451. Type locality: Juan Fernández Islands.

The most common of rockfishes collected on all islands, is distinguished from other species in having mottled anal and caudal fins, 12 dorsal fin spines, 5 branched pectoral fin rays, cheek with ctenoid and cycloid scales, and lacrimal bone with 2-3 prominent spines pointed dorsomedially towards nasal spine. Suborbital sensory canal has skincovered openings with multiple perforations rather than the single pore opening. Coloration and pigmentation pattern are very variable, from red to dark blood-red brown. Records of Scorpaena histrio, though listed by Sepúlveda (1987) and Pequeño \& Sáez (2000), are most probably a mis-identification of this or the following species.

\section{Scorpaena thomsoni Günther, 1880. Fig. 4.}

Scorpaena thomsoni Günther 1880:24, pl. XII. Type locality: Juan Fernández Islands.

Scorpaena uncinata de Buen 1961:32. Type locality: San Ambrosio Island.

The largest of the rockfishes, with 12 dorsal fin spines, last dorsal ray attached by membrane to midline, 6-7 branched pectoral fin rays, no scales on cheek, and dorsomedial spines of lacrimal bone absent or greatly reduced to a nub. The suborbital sensory canal is with normal singlepore openings, sometimes accompanied by two smaller perforations alongside the main pore. Large black blotch on posterior end of spinous dorsal fin is a distinguishing feature when present. Found in Desventuradas and Juan Fernández Islands.

Scorpaenodes englerti Eschmeyer \& Allen, 1971. Fig. 4.

Scorpaenodes englerti Eschmeyer \& Allen 1971:525. Type locality: Tidepools between Hanga Roa \& Hanga Piko, Easter Island.

Similar in size and collected with Scorpaena fernandeziana, but distinguished by lacking the mottled caudal and anal fins, with 13 dorsal fin spines, 8-9 branched pectoral fin rays, ctenoid scales only on cheek, suborbital with reduced spination, nasal spine reduced, lacrimal greatly overlapping maxilla with an undulatory ventral margin ending posteriorly with a blunt spine and large fleshy flap. First record of this species in the Desventuradas Islands (FMNH 109190-109192, MNHNC P7170, 7188, 7196).

\section{NEOSEBASTIDAE}

Scorpionfishes / Escorpénido de arena

Maxillicosta reticulata (De Buen, 1961). Fig. 4.

Cristula reticulata De Buen 1961:27. Type locality: Robinson Crusoe Island, Juan Fernández Islands.

This species is found on sandy bottoms into which they dig backwards, sometimes with only the head in view. Ventral half of body unpigmented, dorsal half with a reticulated pigmentation pattern, dorsal fin mottled and caudal fin banded. Eye large, dorsally flattened with a fringed dorsal margin of pupil. Dorsal fin with 13 spines and 8 soft rays, cheek and body scales cycloid, varying number of spinoid scales behind head and dorsal to lateral line, interorbital space very narrow, numerous small suborbital and supraorbital spines of equal size, maxilla with 3-5 longitudinal ridges. This is the first record of this species for the Desventuradas Islands (FMNH 107372-107375).

\section{TRIGLIDAE}

Gurnards, Searobins / Pez Mariposa

Pterygotrigla picta (Günther, 1880). Fig. 4.

Trigla picta Günther 1880:24. Type locality: Juan Fernández Islands.

Trigla guttata Philippi 1896:375. Type locality: Juan Fernández Islands.

Specimens found in Juan Fernández Islands, in Easter Island and Salas \& Gómez Island. No specimens were found recorded from Desventuradas Islands as mentioned by Pequeño \& Lamilla (2000). Also recorded in southern Australia, New Caledonia, and New Zealand (Kuiter 1993:113). Found at depths of $60 \mathrm{~m}$ (Hubbs 1959) to 320 m (Golovan \& Pakhorukov 1987). De Buen (1959b) provided a detailed description of a single specimen from Robinson Crusoe Island.

\section{POLYPRIONIDAE}

Sea Bass / Bacalao de Juan Fernández

Polyprion oxygeneios (Schneider \& Forster in Bloch \& Schneider, 1801).

Epinephelus oxygeneios Schneider \& Forster in Bloch \& Schneider 1801:301. Type locality: Queen Charlotte Island, New Zealand. 
Polyprion knerii, Steindachner 1875:443. Type locality:

St. Paul's Island; Juan Fernández Islands.

Perca prognatha Forster in Bloch \& Schneider 1801:301. Not available.

Polyprion yanezi De Buen 1959:85. Type locality: 1.5 mi. West of Montemar, Chile.

A deep water species present in seamounts around the Juan Fernández and Desventuradas Islands, as well as in southern Australia, New Zealand, and Tristan da Cunha. Severely overfished.

\section{CALLANTHIIDAE}

Callanthias platei Steindachner, 1898. Fig. 5.

Callanthias platei Steindachner 1898:284. Type locality: Juan Fernández Islands.

Redish with purple hues on head, sometimes with filaments trailing from tips of caudal fin. A beautiful but not very abundant fish, found in both island groups. A species from the Nazca Ridge, Callanthias parini Anderson \& Johnson (1984) is potentially found at Desventuradas Islands at depths over $300 \mathrm{~m}$.

\section{SERRANIDAE}

Sea Basses / Serránidos

Caprodon longimanus Günther, 1859. Fig. 5.

Anthias longimanus Günther 1859:94. Type locality: Indian or Australian Seas

Scorpis fairchildi Hector 1875:241. Type locality: Bay of Plenty, New Zealand.

Neoanthias guntheri Castelnau 1879:367. Type locality: Port Jackson, NSW Australia.

Quite common in Juan Fernández and Desventuradas Islands, it is found usually in small schools of 5 to 10 individuals, though sometimes larger. It is easily recognizable by its long pectoral fin which reaches beyond the origin of the anal fin. It has a broad dark-red lateral stripe from caudal peduncle to mid-body, but which looses definition in the pectoral-postorbital region. Its abundant gillrakers and stomach contents characterize it as an opportunistic pelagic polyphagic-zooplanktivorous predator (Rojas et al. 1998a, c).

Cabrilla

Hypoplectrodes semicinctum (Valenciennes in Cuvier \& Valenciennes, 1833). Fig. 5.
Plectropoma semicinctum Valenciennes in Cuvier \& Valenciennes 1833:442. Type locality: Juan Fernández Islands.

Scopularia rubra De Buen 1959:95. Type locality: Cumberland Bay, Robinson Crusoe Island, Juan Fernández Islands.

Possibly the most ubiquitious of all serranids on these islands. The juveniles with more of a fusiform body and striking horizontal vermiculations streaming from the snout area to the opercle, grow into taller bodied adults without clear head vermiculations. Found in both island groups and one specimen collected at Easter Island (Randall et al. 2005)

Plectranthias exsul Heemstra \& Anderson, 1983.

Plectranthias exsul Heemstra \& Anderson 1983:632.

Type locality: Juan Fernández Islands.

Plectranthias lamillai Rojas \& Pequeño, 1998b.

Plectranthias lamillai Rojas \& Pequeño 1998b:48. Type locality: Rey de Bastos Sector, Alejandro Selkirk

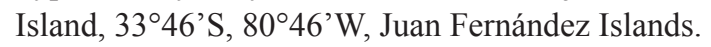

Plectranthias exsul is distributed in Juan Fernández, Desventuradas Islands and Nazca Ridge (Heemstra \& Anderson 1983, Rojas \& Pequeño 1998c, Pequeño \& Lamilla 2000). Plectranthias lamillai is endemic to Alejandro Selkirk Island; however its validity was questioned by Anderson \& Baldwin (2000), based on their comparison of the single type specimen of $P$. lamillai with specimens of similar size and type material of $P$. exsul. The color pattern of $P$. lamillai is different to that of $P$. exsul as characterized by Anderson \& Randall (1991), but Anderson \& Baldwin (2000) considered it "best interpreted as variations on a common theme".

\section{CARANGIDAE}

Jacks / Jurel de Juan Fernández

Pseudocaranx chilensis (Guichenot, 1848). Fig. 5.

Caranx chilensis Guichenot 1848:235. Type locality: Juan Fernández Islands.

This is the predominant pelagic species of the islands, attaining a large size $(650 \mathrm{~mm} \mathrm{TL})$ as in Figure 5 . The horse mackerel, Trachurus murphyi, is often found in Juan Fernández as an incidental catch. Records of Pseudocaranx dentex are considered a misidentification of this species.

Yellowtails / Vidriola, Palometa, Dorado 
Seriola lalandi Valenciennes in Cuvier \& Valenciennes, 1833. Fig. 5.

Seriola lalandi Valenciennes in Cuvier \& Valenciennes 1833:208. Type locality: Brazil.

Seriola aureovittata Temminck \& Schlegel 1845:115. Type locality: Japan.

Seriola banisteri Smith 1959:256. Type locality: Off Hibberdene, Natal, South Africa.

Seriola (Buphthalmus) bovinoculata Smith 1959:259. Type locality: Pinda, Mozambique.

Halatractus dorsalis Gill 1863:84. Type locality: Cape San Lucas, Baja California, México.

Seriola foncki Delfin 1903:222. Type locality: Juan Fernández Islands.

Seriola grandis Castelnau 1872:115. Type locality: Melbourne market, Victoria, Australia.

Seriola mazatlana Steindachner 1876:56. Type locality: Mazatlán, Sinaloa, w. México.

Lichia pappei Bleeker (ex Castelnau) 1860:65. Type locality: Kalk Bay, South Africa.

This pelagic species is readily found around the shores of Juan Fernández Islands during the spring and summer months (Arana 2000) in schools of varying sizes. It is highly regarded by fishermen which draws an incipient alternative business to the islands.

\section{SCIAENIDAE}

Grunts / Corvina de Juan Fernández

Umbrina reedi Günther, 1880. Fig. 5.

Umbrina reedi Günther 1880:25. Type locality: Juan Fernández Islands.

Endemic to both island groups (Kong \& Valdés 1990, Walker \& Radford 1992). The scale row pigmentation pattern -horizontal below lateral line and transversal above lateral line- is less evident in larger specimens.

\section{KYPHOSIDAE}

Sea Chubs /Vieja negra, Jerguilla de Juan Fernández

Girella albostriata Steindachner, 1898. Fig. 5.

Girella albostriata Steindachner 1898:287. Type locality: Juan Fernández Islands.

Girella feliciana Clark 1938:180. Type locality: Tide pools, San Félix Island.
Endemic to both island groups. Large herbivorous fishes (Duhart \& Ramírez 1998) with a juvenile phase in tide pools. We regard G. feliciana to represent the juvenile intertidal pool-resident of $G$. albostriata. The thin light vertical striations over a black-green body that characterize this species are variable in number and visibility, are more clearly visible underwater and fade quickly after being fished. The northern Pacific Girella nigricans was cited for Juan Fernández Islands by Pequeño \& Sáez (2000), though not on a specimen-based identification.

Sweeps / Pampanito de Juan Fernández

Scorpis chilensis Guichenot, 1848. Fig. 5.

Scorpis chilensis Guichenot 1848:220. Type locality: Juan Fernández Islands.

Endemic to both island groups and very abundant. Single southeastern Pacific species with other four congeneric species inhabiting Australian and New Zealand waters.

\section{CHAETODONTIDAE}

Narrow-barred butterflyfish / Pez mariposa de Juan Fernández

Amphichaetodon melbae Burgess \& Caldwell in Burgess, 1978. Fig. 5.

Chaetodon aureus Molina 1782:127.

Chaetodon chilensis Gmelin 1789:1264.

Chaetodon chilensis Molina 1810:193.

Amphichaetodon melbae Burgess \& Caldwell in Burgess 1978:291. Type locality: San Félix Island.

Found in both island groups and Easter Island. The descriptions provided by Molina $(1782,1810)$ clearly refer to this species, but as the names aureus or chilensis have never before been associated with this species, we have opted to maintain the name melbae for the purpose of taxonomic stability until a proper taxonomic revision of the species has been undertaken.

\section{CHIRONEMIDAE}

Kelpfishes /Yunyungo, Cachudito.

Chironemus bicornis (Steindachner, 1898). Fig. 6.

Chilodactylus bicornis Steindachner 1898:291. Type locality: Juan Fernández Islands. 

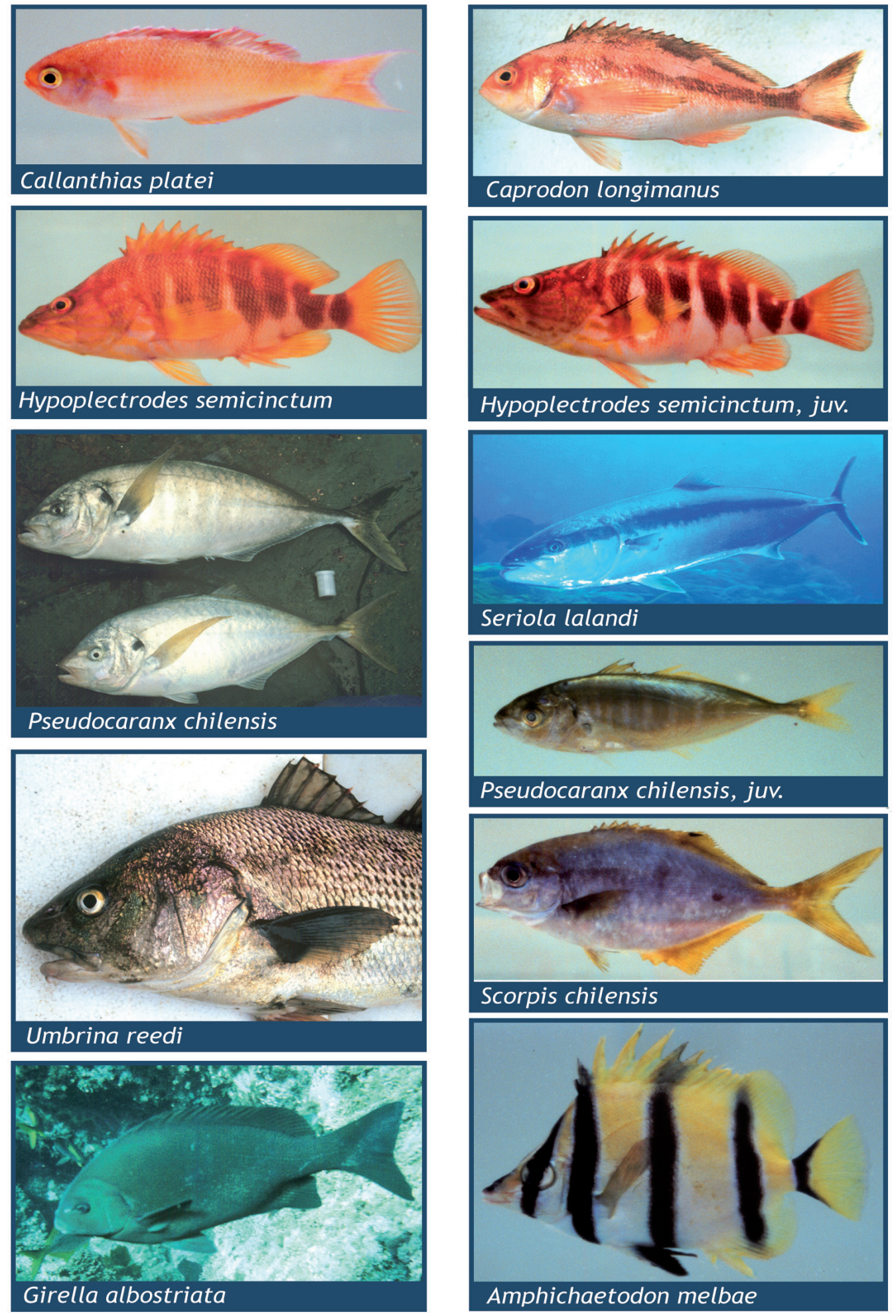

Figure 5. Species of the fish families Callanthiidae, Serranidae, Carangidae, Sciaenidae, Kyphosidae, and Chaetodontidae. Underwater photos of S. lalandi and G. albostriata by M. Duhart / Especies de peces de las familias Callanthiidae, Serranidae, Carangidae, Sciaenidae, Kyphosidae y Chaetodontidae. Fotos submarinas de S. lalandi y $G$. albostriata por M. Duhart 
Present in both island groups. Relatively abundant, especially within the $5 \mathrm{~m}$ depth range. Found perched with pectoral fins on rocks at different angles where they eat mostly small invertebrates.

Kelpfishes / Cabrilla de Juan Fernández

Chironemus delfini (Porter, 1914). Fig. 6.

Chilodactylus delfini Porter 1914:204. Type locality: Juan Fernández Islands.

Present on Juan Fernández islands only (Meléndez \& Dyer 2010). Less abundant than Ch. bicornis, fully sympatric, but not sister species (Burridge et al. 2006). Found under large boulders, slightly territorial and not aggresive.

\section{CHEILODACTYLIDAE}

Morwongs / Breca

Nemadactylus gayi (Kner 1865). Fig. 6.

Cheilodactylus gayi Kner 1865:92. Type locality: Chile.

Present in both island groups. Previously placed in Acantholatris or Cheilodactylus, is now referred to Nemadactylus according to Burridge (1999a,b). Presence of this species in southern continental America is questionable (Burridge 1999b) and most probably is based on misidentifications of Nemadactylus bergi (Pequeño 2004).

\section{LABRIDAE}

Wrasses / Vieja de Juan Fernández

Pseudolabrus gayi (Valenciennes in Cuvier \& Valenciennes, 1839). Fig. 6.

Labrus gayi Valenciennes in Cuvier \& Valenciennes 1839:97. Type locality: Juan Fernández Islands.

The species is present abundantly in both island groups (Russell 1988). It is distinguished from other wrasses by a shorter and deeper body. A horizontal line passing over the pectoral fin axilla divides a darker dorsal half from a lighter to silvery ventral area.

Wrasses / Vieja, Biya

Malapterus reticulatus Valenciennes in Cuvier \& Valenciennes, 1839. Fig. 6.

Malapterus reticulatus Valenciennes in Cuvier \& Valenciennes 1839:355. Type locality: Juan Fernández Islands.

Neolabrus fenestratus Steindachner 1875:461. Type locality: Juan Fernández Islands.
The most abundant species together with Scartichthys and Pseudolabrus. With their characteristic pectoralfin locomotor pattern, individuals peck-feed at small invertebrates and flock around ready to get whatever is kicked up by the diver. This species has two adult color patterns, the green-yellow phase and the black phase (Fig. 6). Juveniles display a black blotch on the anterior half of the soft dorsal fin (Fig. 6) which later disappears, leaving only the anterior blotch over the first three dorsal fin spines. They are also known to be cleaners of Scorpis individuals, feeding off buccal isopod ectoparasites.

Suezichthys sp. Russell \& Westneat (in prep.) Fig. 6.

Type locality: Robinson Crusoe Island, SE of Bacalao Point (33³8'20”S; 7847'15’W).

Present on both island groups and a range extension for the genus. It is distiguished from its family relatives on the islands by a large blotch extended over four or five dorsal fin rays, over the abdominal region and seven or eight saddled bands over the dorsal half of the body.

\section{POMACENTRIDAE}

Damselfishes / Castañeta

Chromis meridiana Greenfield \& Woods, 1980. Fig. 7.

Chromis meridiana Greenfield \& Woods 1980:634. Type locality: San Félix Island.

The only damselfish species around the Desventuradas Islands, distinguished by a white patch saddled immediately posterior to the soft dorsal fin, 17 tubed scales on upper lateral line, 14 dorsal spines, and two dorsal and two ventral procurrent caudal fin spines. Chromis crusma has been recorded in Juan Fernández by numerous authors (De Buen 1959a, Pequeño \& Sáez 2000) but these seem to be errors as none have been collected (Pequeño et al. 2005).

\section{PINGUIPEDIDAE}

Grubfishes / Rollizo de Juan Fernández

Parapercis dockinsi McCosker, 1971. Fig. 7.

Parapercis dockinsi McCosker 1971:682. Type locality: $1 \mathrm{~km}$ northwest of San Carlos Point, Juan Fernández Islands, off Chile,

Present usually on whitish sandy bottoms where it is easily camouflaged. Endemic to the Juan Fernández Islands.

\section{BLENNIIDAE}

Blennies / Cachudito o Torito de Juan Fernández

Scartichthys variolatus (Valenciennes in Cuvier \& Valenciennes, 1836) Fig. 7. 


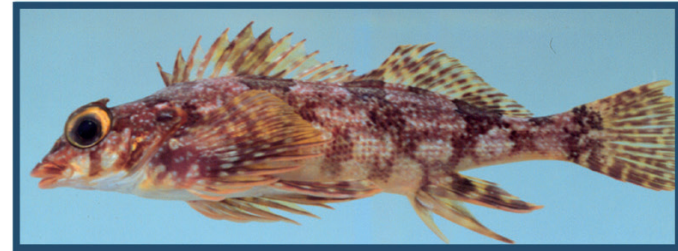

Chironemus bicornis
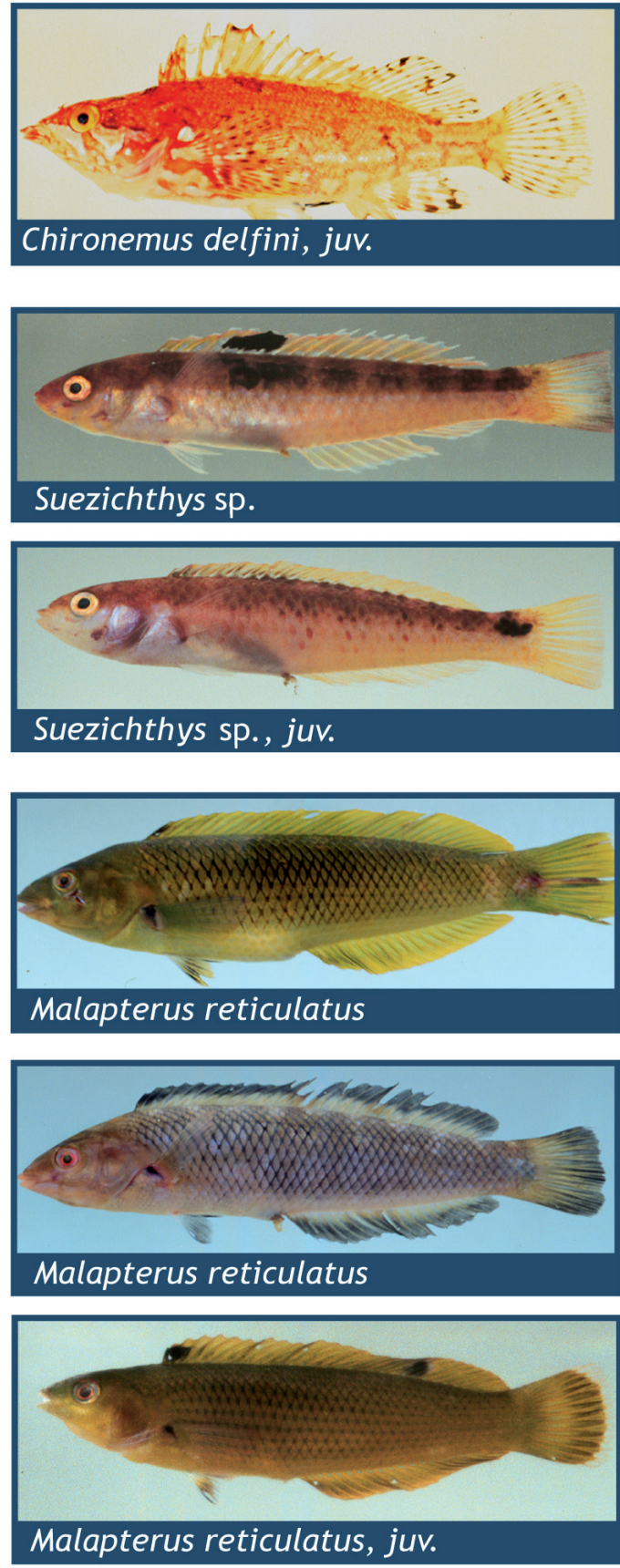

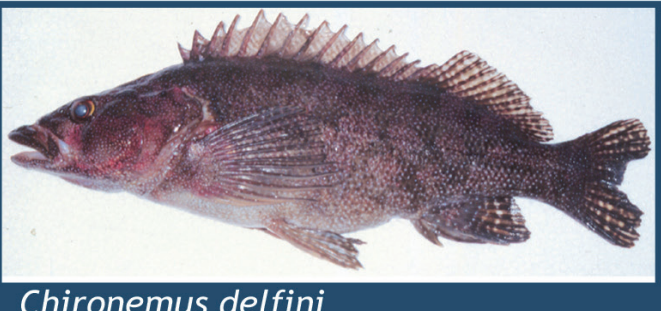

\section{Chironemus delfini}
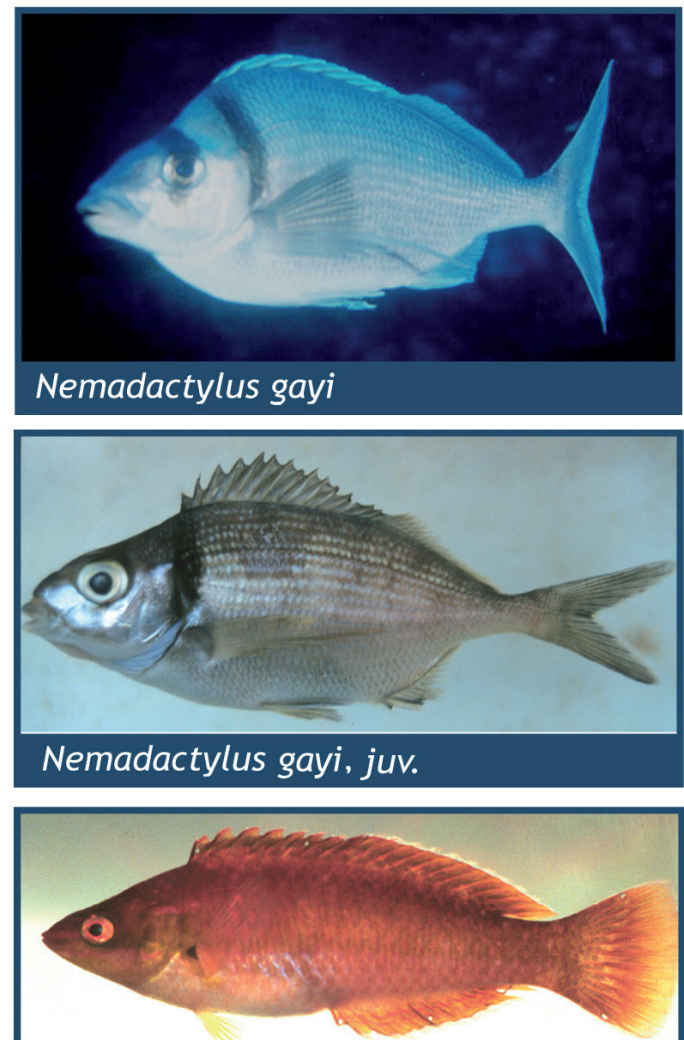

Pseudolabrus gayi
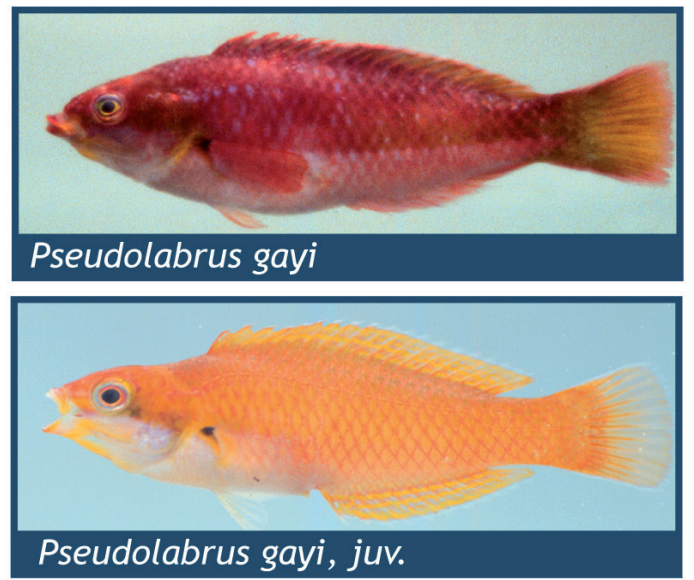

Figura 6. Species of the fish families Chironemidae, Cheilodactylidae, and Labridae. Underwater photo of N. gayi by A. Sepúlveda / Especies de peces de las familias Chironemidae, Cheilodactylidae y Labridae. Foto submarina de $N$. gayi por A. Sepúlveda 
Salarias variolatus Valenciennes in Cuvier \& Valenciennes 1836:346. Type locality: Juan Fernández Islands.

Salarias rubropunctatus Valenciennes in Cuvier \& Valenciennes 1836:348. Type locality: Juan Fernández Islands.

Ophioblennius fernandezensis Clark 1938:184. Type locality: San Juan Bautista (Cumberland) Bay, Juan Fernández Islands.

Salarias cuvieri Günther 1861:248. Type locality: Chile.

The most abundant species on the rocky shores of both island groups, wherever there are algae. It displays a diverse range of color patterns according to age and location, but all with the characteristic red spots over the head and body, and the black blotch between the first two rays of the dorsal fin. Premetamorphic individuals collected at intertidal pools in Robinson Crusoe Island are semi-transparent and display canine-like teeth. Adult males with the first three rays of the anal fin enlarged and swollen. Scartichthys viridis has been reported in Robinson Crusoe based on a single specimen (MNHNC P6753, Meléndez \& Villalba 1992) and considered incidental.

Entomacrodus chapmani Springer, 1967.

Entomacrodus chapmani Springer 1967:95. Type locality: Easter Island, SE Pacific.

Though not collected in this expedition, a specimen was collected at San Félix (IZUA 1478, Pequeño \& Lamilla 2000, Pequeño \& Sáez 2004).

\section{GOBIIDAE}

Gobies / Pez neón

Paratrimma nigrimenta Hoese \& Brothers, 1976. Fig. 7.

Paratrimma nigrimenta Hoese \& Brothers 1976:496. Type locality: $1 \mathrm{~km}$ northwest of San Carlos, Juan Fernández Islands.

Paratrimma urospila Hoese \& Brothers, 1976.

Paratrimma urospila Hoese \& Brothers 1976:497. Type locality: NW side of San Félix Island.

Only P. nigrimenta was collected at Robinson Crusoe and Alejandro Selkirk Islands, in or at the mouth of small caves and crevices, down to $30 \mathrm{~m}$. Bright yellow body with purple snout and forehead, tapering posteriorly to the base of the first dorsal fin, which has an extended first ray in males.

\section{PARALICHTHYIDAE}

Flatfishes/ Lenguados

Paralichthys fernandezianus Steindachner, 1903. Fig. 7.

Paralichthys fernandezianus Steindachner 1903:208. Type locality: Juan Fernández Islands.

Paralichthys hilgendorfii Steindachner 1903:209. Type locality: Juan Fernández Islands.

Paralichthys schmitti Ginsburg 1933:1. Type locality: Juan Fernández Islands.

Snout naked. Blind side with preopercle, mandible and maxilla naked. Eyed side with ctenoid scales whereas blind side with scales cycloid. Interorbital space one third to half the vertical eye diameter. Presence of blotches on the blind side of dorsal and anal fins, and pigment between the lower mandible and the pelvic fin of the blind side. Mandibles with sharp canine-like teeth. Present in Juan Fernández and Desventuradas Islands groups.

Arnoglossus coeruleosticta (Steindachner, 1898). Fig. 7.

Paralichthys coeruleosticta Steindachner 1898:327. Type locality: Juan Fernández Islands.

Snout with scales. Preopercle, mandible and maxilla of blind side with scales. Interorbital space narrow, less than one third the vertical eye diameter. Mandibles with a single row of fine teeth. Present in Juan Fernández and Desventuradas Islands groups.

\section{SOLEIDAE}

Soles / Lenguado

Aseraggodes bahamondei Randall \& Meléndez, 1987. Fig. 7.

Aseraggodes bahamondei Randall \& Meléndez 1987:99. Type locality: Off Mataveri O Tai near S end of W coast, Easter Island.

This species was collected with bottom trawl off San Félix, extending its geographic distribution across the Pacific from Lord Howe and Norfolk to Desventuradas Islands.

\section{BiogeOgRAPHY}

Pequeño \& Lamilla (1996a, 2000) supported Parin's (1991) proposal of extending the Indo-West Pacific zoogeographic region across to the southeastern Pacific to include not only the Nazca Ridge but also the Desventuradas Islands. Pequeño \& Sáez (2000) took this one step further and extended it to the Juan Fernández Islands. Pequeño \& 

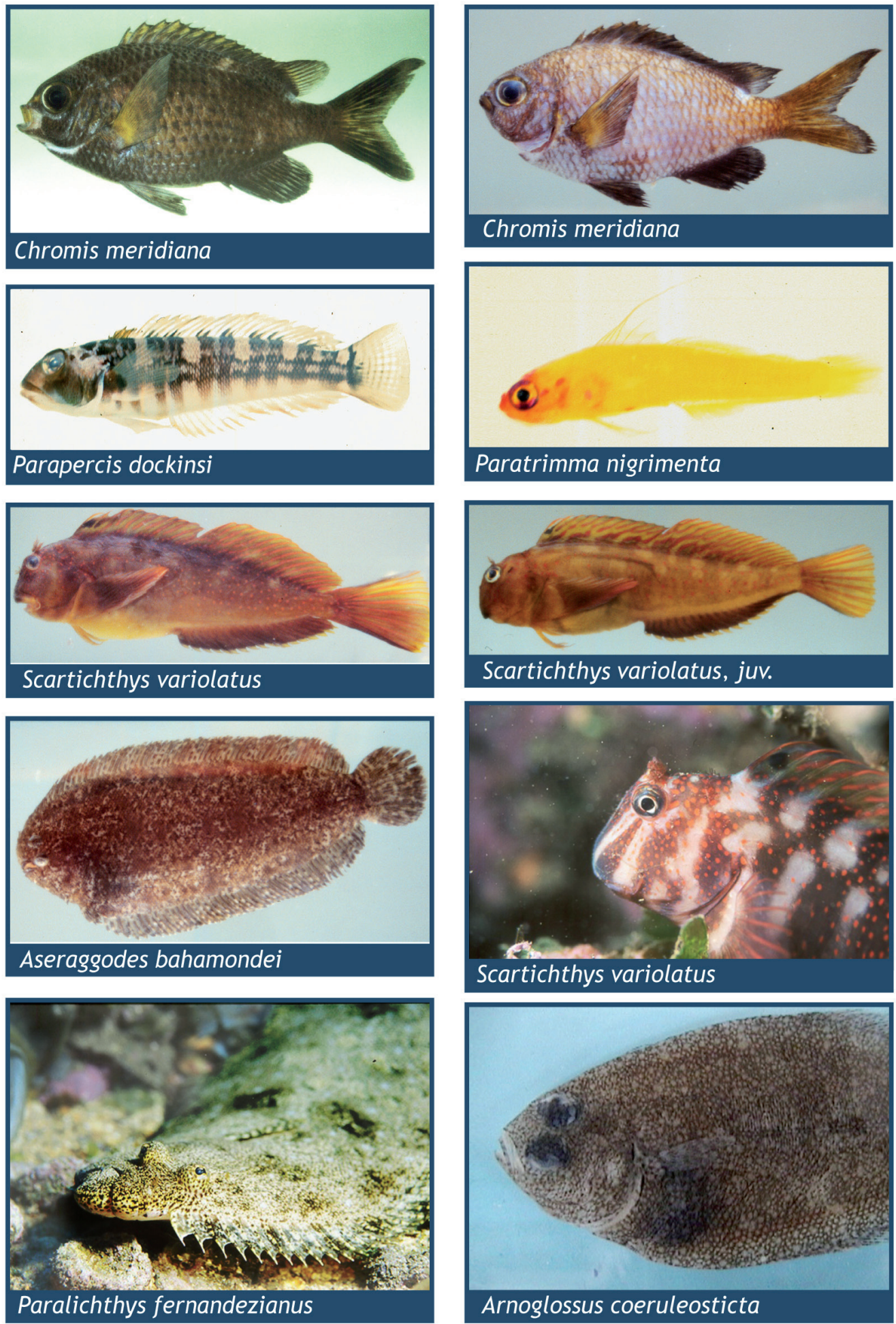

Figure 7. Species of the fish families Pomacentridae, Pinguipedidae, Gobiidae, Blenniidae, Soleidae, and Paralichthyidae. Underwater photos of S. variolatus and P. fernandezianus by A. Sepúlveda and M. Rossi / Especies de peces de las familias Pomacentridae, Pinguipedidae, Gobiidae, Blenniidae, Soleidae y Paralichthyidae. Fotos submarinas de S. variolatus y P. fernandezianus por A. Sepúlveda y M. Rossi 
Lamilla (2000) reviewed the zoogeographical affinities between Desventuradas and the western Pacific islands (Easter Island to Australia), increasing the number of shared species from eight to fifteen (Table 3). This paper supplies an additional five species to their list: Gymnothorax cf. obesus, Gonorynchus greyi, Antennarius sanguineus, Scorpaenodes englerti, and Aseraggodes bahamondei.

The number of species shared between two regions (bioaccountancy of Greenwood 1983) is a measure of recent or present-day connectivity between the regions, however, says nothing of the past relations (historical biogeography), an issue overlooked by previous biogeographical studies of these island groups (Pequeño \& Lamilla 1996a, 2000, Pequeño \& Sáez 2000). Three phylogenetic studies exist to date at the intrageneric level that include fishes from these islands: Pseudolabrus (Russell 1988), Nemadactylus (Burridge 1999b), and Chironemus (Burridge et al. 2006). All these studies indicate a West Pacific origin of the genera, with one or multiple dispersal events towards the East.

In the absence of a phylogenetic study, however, the hypothesis of a shared historical relationship inherently contained in the congeneric membership of two or more species, provides a measure of historical relationships between the areas inhabited by the species involved, especially when the genus is clearly monophyletic, derived and relatively restricted geographically. Genera that seem to support the above pattern of historical relationships are: Lotella, Paratrachichthys, Monocentris, Scorpis, Amphichaetodon, Maxillicosta, and Suezichthys. Knowledge of the species relationships within these genera would provide the required support for a more general statement of the historical biogeography of the fishes of the southern Pacific Ocean.

Though some species are shared with the continent (see Table 3), some species also show a historical relationship with the continent, either by phylogenetic analysis (Odontesthes gracilis Dyer 1998, 2000, Dyer \& Gosztonyi 1999) or by having their closest relatives only on continental America as is the case with Scartichthys variolatus, Ophidion metoecus, and Sicyases brevirostris.

The Desventuradas and Juan Fernández Islands also share a fish fauna that is endemic to these islands as a geographic unit. Pequeño \& Lamilla (2000:433) reported 21 species endemic to these two island groups, whereas Pequeño \& Sáez (2000:30) indicated that 15 species were shared between them. This work reports a total 22 species endemic to both island groups as a geographic unit (Scolecenchelys chilensis, Lotella fernandeziana, Ophidium metoecus, Paratrachichthys fernandezianus, Cosmocampus arctus heraldi, Scorpaena fernandeziana, Scorpaena thomsoni, Maxillicosta reticulata, Callanthias platei, Hypoplectrodes semicinctum, Plectranthias exsul, Pseudocaranx chilensis, Umbrina reedi, Girella albostriata, Scorpis chilensis, Chironemus bicornis, Nemadactylus gayi, Pseudolabrus gayi, Malapterus reticulatus, Suezichthys sp., Scartichthys variolatus, Paralichthys fernandezianus, Arnoglossus coeruleosticta). Thus the Juan Fernández and Desventuradas Islands together exhibit a $42,3 \%$ endemicity of coastal fishes (over $68 \%$ when considering only species common to both island groups, 32 see Table 3 ) and as such we propose they be considered as a biogeographic unit.

\section{Discussion}

The Juan Fernández and Desventuradas Islands have a total of 52 species of coastal fishes, with 41 and 43 species in each, respectively. The number of endemic species for Juan Fernández is down from 13 (25.5\%; Pequeño \& Sáez 2000) to 5 (12.2\%; Sicyases brevirostris, Odontesthes gracilis, Chironemus delfini, Parapercis dockinsi, and Paratrimma nigrimenta), however three species are presented as new records for these islands (Gymnothorax cf. obesus, Gnathophis sp. and Suezichthys $\mathrm{sp})$. The Desventuradas Islands has only 2 endemic species (4.6\%; Chromis meridiana and Paratrimma urospila) but 4 species are new records (Scorpaenodes englerti, Maxillicosta reticulata, Suezichthys sp., and Aseraggodes bahamondei).

The eastern extension of the Indo West Pacific Region to include the Nazca Ridge, Desventuradas and Juan Fernández Islands (Parin 1991, Pequeño \& Lamilla 1996a, 2000, Pequeño \& Sáez 2000) is supported in this paper. The zoogeographical affinities between Desventuradas and western Pacific islands (Easter Island to Australia), includes 16 species, adding 4 new species to previous lists (Gymnothorax cf. obesus, Gonorynchus greyi, Scorpaenodes englerti, Aseraggodes bahamondei). Three phylogenetic studies (Pseudolabrus, Nemadactylus, and Chironemus) indicate a Western Pacific origin with one or more dispersal events to the Desventuradas and Juan Fernández Islands, and only one (Odontesthes) indicates a continental origin with a westward dispersal. The pattern of historical relationships of the Desventuradas and Juan Fernández Islands with the Western Pacific is supported with another 7 genera with relatively few species and 
restricted to the southern Pacific region. Furthermore, the Juan Fernández and Desventuradas Islands share 22 coastal fish species that are endemic to those island groups alone (42,3\%), suggesting these two island groups should be considered as a single biogeographic unit.

\section{ACKnowledgments}

We wish to thank the National Geographic Foundation grant 5257-96 that made this cruise possible, to Siegfried Ziller that turned the logistic nightmare into a successful 23-day cruise, a record for the aging M/V "Carlos Porter" made available to us by the Instituto Fomento Pesquero (IFOP). We also wish to thank Marcel Duhart and Daniel Elton for their important contribution in diving and trawling collections. We especially thank Marcel Duhart and Álvaro Sepúlveda ${ }^{2}$ for allowing us to use their splendid underwater photographs. This work was also supported by NSF grant DEB-0844745 and funding from the John D. and Catherine T. MacArthur Foundation for the Encyclopedia of Life.

I (BSD) wish to thank the Field Museum's Robert O. Bass Visiting Scientist Fund for a month's visit in Chicago in 2000. We wish to thank the following for loans of specimens to FMNH: Karsten Hartel (MCZ), John Friel (CU), and H.J. Walker (SIO). Additional material (fishes, photos, tissues) was made available thanks to FONDECYT grant $N^{\circ} 1990172$ to R. Meléndez \& B.S. Dyer, and the incredible support received by Marcelo Rossi and "Peter" Niada from ENDEMICA Expeditions on Robinson Crusoe and Alejandro Selkirk Islands.

\section{LITERATURE CITED}

Anderson WD Jr. \& CC Baldwin. 2000. A new species of Anthias (Teleostei: Serranidae: Anthiinae) from the Galápagos Islands, with keys to Anthias and eastern Pacific Anthiinae. Proceedings of the Biological Society of Washington 113(2): 369-385.

Anderson WD Jr. \& GD Johnson. 1984. A new species of Callanthias (Pisces: Perciformes: Percoidei: Callanthiidae) from the southeastern Pacific Ocean. Proceedings of the Biological Society of Washington 97(4): 942-950.

Anderson WD Jr. \& JE Randall. 1991. A new species of the anthiine genus Plectranthias (Pisces: Serranidae) from the Sala y Gómez Ridge in the eastern South Pacific, with comments on P. exsul. Proceedings of the Biological Society of Washington 104(2): 335-343.

Arana P. 1987. Perspectivas históricas y proyecciones de la actividad pesquera realizada en el Archipiélago de Juan Fernández, Chile. In: Castilla JC (ed). Islas oceánicas chilenas: conocimiento científico y necesidades de investigaciones, pp. 319-353. Ediciones Universidad Católica de Chile, Santiago.

Arana PM. 2000. Experiencias de pesca con red de enmalle en las islas Robinson Crusoe y Santa Clara, Chile. Investigaciones Marinas 28: 231-237.

Bleeker P. 1866. Sur les espèces d'Exocet de l'Inde Archipélagique. Nederlandsch Tijdschrift voor de Dierkunde 3: 105-129.

Bloch ME \& JG Schneider. 1801. M. E. Blochii, Systema Ichthyologiae iconibus cx illustratum. Post obitum auctoris opus inchoatum absolvit, correxit, interpolavit Jo. Gottlob Schneider, Saxo. Berolini. Systema Ichthyologiae: 1-584. Sumtibus Austoris Impressum et Bibliopolio Sanderiano Commissum.

Briggs JC. 1955. A monograph of the clingfishes (Order Xenopterygii). Stanford Ichthyological Bulletin 6: 1-224.

Burgess WE. 1978. Butterflyfishes of the world. A monograph of the family Chaetodontidae, 832 pp. T.F.H. Publications, Neptune City.

Burridge CP.1999a. Suggestion of synonymy for Nemadactylus and Acantholatris (Perciformes: Cirrhitoidea). In: Séret B \& J-Y Sire (eds). Proceedings of the 5th Indo-Pacific Fish Conference, Noumea, 1997, pp. 413-416.

Burridge CP. 1999b. Molecular phylogeny of Nemadactylus and Acantholatris (Perciformes: Cirrhitoidea), with implications for taxonomy and biogeography. Molecular Phylogenetics and Evolution 13: 93-109.

Burridge CP, R Meléndez \& BS Dyer. 2006. Multiple origins of the Juan Fernández kelpfish fauna and evidence for frequent and unidirectional dispersal of Cirrhitoid fishes across the South Pacific. Systematic Biologist 55(4): 566578.

Castilla JC \& D Oliva. 1987. Islas oceánicas chilenas: aspectos descriptivos y potencialidades. In: Castilla JC (ed). Islas oceánicas chilenas: conocimiento científico y necesidades de investigaciones, pp. 15-35. Ediciones Universidad Católica de Chile, Santiago.

Castle PHJ \& JE McCosker. 1999. A new genus and two new species of myrophine worm-eels, with comments on Muraenichthys and Scolecenchelys (Anguilliformes: Ophichthidae). Records of the Australian Museum 51: 113-122.

Clark HW. 1938. Additional new fishes. The Templeton Crocker Expedition of 1934-35. No. 36. Proceedings of the California Academy of Sciences 22(7): 179-185.

Compagno LJV. 1984. FAO species catalogue. Vol. 4. Sharks of the world. An annotated and illustrated catalogue of shark species known to date. Part 1 - Hexanchiformes to Lamniformes, 249 pp. FAO, Roma.

Cuevas JG \& G Van Leersum. 2001. Project “Conservation, restoration, and development of the Juan Fernández Islands, Chile". Revista Chilena de Historia Natural 74: 899-910. 
Cuvier G \& A Valenciennes. 1833. Histoire naturelle des poissons. Tome neuvième. Suite du livre neuvième. Des Scombéroïdes. Histoire Naturelle des Poissons 9: 1-512.

Cuvier G \& A Valenciennes. 1836. Histoire naturelle des poissons. Tome onzième. Livre treizième. De la famille des Mugiloïdes. Livre quatorzième. De la famille des Gobioïdes. Histoire Naturelle des Poissons 11: 1-506.

Cuvier G \& A Valenciennes. 1839. Histoire naturelle des poissons. Tome treizième. Livre seizième. Des Labroïdes. Histoire Naturelle des Poissons 13: 1-505

De Buen F. 1959a. Lampreas, tiburones, rayas y peces en la Estación de Biología Marina de Montemar, Chile. (Primera contribución). Revista de Biología Marina 9(1-3): 3-200.

De Buen F. 1959b. Notas sobre ictiología chilena, con descripción de dos especies nuevas. Revista de Biología Marina 9(1-3): 257-270.

De Buen F. 1960. Los peje-sapos (Familia Gobiesocidae) en Chile. Revista de Biología Marina 10(1-3): 69-82

De Buen F. 1961. Peces chilenos. Familias Alepocephalidae, Muraenidae, Sciaenidae, Scorpaenidae, Liparidae y Bothidae. Montemar 1: 1-52. [Revista de Biología Marina 11]

De Buen F. 1963. Peces chilenos: Beloniformes, Syngnathiformes y Gobiidae. Boletín de la Sociedad de Biología de Concepción, Chile 35-36: 81-101.

Delfin FT. 1899. Nuevo pez para la fauna de Chile. Revista Chilena de Historia Natural 3: 75-78.

Di Salvo L, J Randall \& A Cea. 1988. Ecological reconnaissance of the Easter Island sublittoral marine environment. National Geographic Research 4(4): 451-473.

Duhamel G. 1995. Révision des genres Centriscops et Notopogon, Macroramphosidae des zones subtropicale et tempérée de l'hémisphère sud. Cybium 19(3): 261-303.

Duhart M \& M Ramírez. 1998. Análisis trófico de Girella feliciana Clark, 1938, y Girella albostriata Steindachner, 1898, presentes en los archipiélagos Desventuradas y Juan Fernández (Osteichthyes, Perciformes, Girellidae). Noticiario Mensual, Museo Nacional de Historia Natural 333: 8-12.

Dyer BS. 1998. Phylogenetic systematics and historical biogeography of the Neotropical silverside family Atherinopsidae (Teleostei: Atheriniformes). In: Malabarba LR, RE Reis, RP Vari, ZM Lucena \& CAS Lucena (eds). Phylogeny and classification of neotropical fishes, pp. 519-536. Edipucrs, Porto Alegre.

Dyer BS. 2000. Revisión sistemática de los pejerreyes de Chile (Teleostei, Atheriniformes). Estudios Oceanológicos 19: 99-127.

Dyer BS \& A Gosztonyi. 1999. Phylogenetic revision of the South American subgenus Austromenidia Hubbs, 1918 (Teleostei, Atherinopsidae, Odontesthes) and a study of meristic variation. Revista de Biología Marina y Oceanografía 34(2): 211-232.

Eschmeyer WN. 2010. Catalog of fishes, electronic version. [on-line]<http://research.calacademy.org/ichthyology/ catalog/fishcatmain.asp $>$
Eschmeyer WN \& GR Allen. 1971. Three new species of scorpionfishes (family Scorpaenidae) from Easter Island. Proceedings of the California Academy of Sciences 37(19): 515-527.

Eschmeyer WN \& S Poss. 1976. Review of the scorpionfish genus Maxillicosta (Pisces: Scorpaenidae), with a description of three new species from the Australian-New Zealand region. Bulletin of Marine Sciences 26(4): 433449.

Fritzsche RA. 1980. Revision of the eastern Pacific Syngnathidae (Pisces: Syngnathiformes), including both recent and fossil forms. Proceedings of the California Academy of Sciences 42(6): 181-227.

Gill TN. 1863. Descriptions of some new species of Pediculati, and on the classification of the group. Proceedings of the Academy of Natural Sciences of Philadelphia 15: 88-92.

Ginsburg I. 1933. Descriptions of new and imperfectly known species and genera of gobioid and pleuronectid fishes in the United States National Museum. Proceedings of the United States National Museum 82 (2961): 1-23.

Golovan A \& N Pakhorukov. 1987. Distribution and behavior of fishes on the Nazka and Sala y Gomez submarine ranges. Voprosy Ikhtiologii 3:369-376.[Journal of Ichthyology]

Gomon M, J Glover \& R Kuiter. 1994. The fishes of Australia's South Coast. The flora and fauna of South Australia handbook Committee, 992 pp. State Print, Adelaide.

González-Ferrán O. 1987. Evolución geológica de las islas chilenas en el Océano Pacífico. In: Castilla JC (ed). Islas oceánicas chilenas: conocimiento científico y necesidades de investigaciones, pp. 37-54. Ediciones Universidad Católica de Chile, Santiago.

Grande T. 1999. Revision of the genus Gonorynchus Scopoli, 1777 (Teleostei: Ostariophysi). Copeia 1999(2): 453-469.

Greenfield DW \& LP Woods. 1980. Review of the deepbodied species of Chromis (Pisces: Pomacentridae) from the eastern Pacific, with descriptions of three new species. Copeia 1980(4): 626-641.

Guichenot A. 1848. Fauna chilena. Peces. In: Gay C (ed). Historia física y politica de Chile, Zoologia 2: 137-370. Claudio Gay, Paris \& Santiago.

Günther A. 1859. Catalogue of the acanthopterygian fishes in the collection of the British Museum. I. Gasterosteidae, Berycidae, Percidae, Aphredoderidae, Pristipomatidae, Mullidae, Sparidae. Catalogue of the Fishes in the British Museum 1: 1-524.

Günther A. 1880. Report on the shore fishes procured during the voyage of H. M. S. Challenger in the years 1873-1876. Report of the Scientific Results of the Voyage of H. M. S. Challenger during the years 1873-76, Zoology 1(6): 1-82.

Günther A. 1887. Report on the deep-sea fishes collected by H. M. S. Challenger during the years 1873-76. Report of the Scientific Results of the Voyage of H.M.S. Challenger during the years 1873-76, Zoology 22(57): 1-268. 
Heemstra PC \& WD Anderson Jr. 1983. A new species of the serranid fish genus Plectranthias (Pisces: Perciformes) from the southeastern Pacific Ocean, with comments on the genus Ellerkeldia. Proceedings of the Biological Society of Washington 96(4): 532-637.

Hoese D \& E Brothers. 1976. Paratrimma, a new genus of gobiid fishes and two new species. Copeia 1976(3): 494497.

Hoffman AJ \& C Marticorena. 1987. La vegetación de las islas oceánicas chilenas. In: Castilla JC (ed). Islas oceánicas chilenas: conocimiento científico y necesidades de investigaciones, pp. 127-165. Ediciones Universidad Católica de Chile, Santiago

Hubbs CL. 1959. Initial discoveries of fish faunas on seamounts and offshore banks in the eastern Pacific. Pacific Science 13(4): 311-316.

Jenyns L. 1842. Fish. In: Darwin C (ed). The zoology of the voyage of H. M. S. Beagle, under the command of Captain Fitzroy, R. N., during the years 1832 to 1836 , pp. 1-172 Smith, Elder and Company, London.

Karmovskaya ES. 1990. New species of conger eels from southern Pacific seamounts. Voprosy Ikhtiologii 30(5): 764772. [Journal of Ichthyology]

Karmovskaya ES \& JR Paxton. 2000. Revision of the Australian congrid eels of the genus Gnathophis (Family Congridae), with descriptions of six new species. Journal of Ichthyology 40(Suppl. 1): 1-14.

Kner R. 1865. Fische. Reise der österreichischen Fregatte Novara um die Erde in den Jahren 1857, 1858, 1859, unter den Befehlen des Commodore B. von Wüllerstorf-Urbain. Wien. Novara Expedition, Zoologischer Theil 1(1): 1-109.

Kong I \& J Valdés. 1990. Sciaenidos de Chile: análisis taxonómico y morfológico. Estudios Oceanológicos 9: 13-56.

Kotlyar AN. 1985. Taxonomy and distribution of Monocentridae (Beryciformes). Journal of Ichthyology 25(4): 91-106.

Kotlyar AN. 1996. Beryciform fishes of the world ocean, 368 pp. VNIRO Publishing, Moscow.

Kuiter RH. 1993. Coastal fishes of south-eastern Australia, 437 pp. University of Hawaii Press, Honolulu.

Lavenberg RJ. 1992. A new moray eel (Muraenidae: Gymnothorax) from oceanic islands of the south Pacific. Pacific Science 46: 58-67.

Lesson RP. 1831. Poissons. In: Duperrey LI (ed). Voyage Autour du Monde, exécuté par ordre du Roi, sur la corvette de sa Majesté, La Coquille, pendant les années 1822, 1823, 1824 et 1825 sous les ministère et conformément aux instructions de S.E.M. le Marquis de Clermont-Tonnerre, Ministre de la Marine; et publié sous les auspices de son Excellence Mgr le Cte de Chabrol, Ministre de la Marine et des Colonies. Voyage Coquille Zoologie 2(1): 66-238. A. Bertrand, Paris.

Matthiessen B, HO Fock \& H von Westernhagen. 2003. Evidence for two sympatric species of snipefishes
Macroramphosus spp. (Syngnathiformes, Centriscidae) on Great Meteor Seamount. Helgoland Marine Research 57: 63-72.

McCosker JE. 1970. A review of the eel genera Leptenchelys and Muraenichthys, with the description of a new genus, Schismorhynchus, and a new species Muraenichthys chilensis. Pacific Science 24: 506-516.

McCosker JE. 1971. A new species of Parapercis (Pisces: Mugiloididae) from the Juan Fernández Islands. Copeia 1971(4): 682-686.

McMillan CB \& RL Wisner. 1984. Three new species of seven-gilled hagfishes (Myxinidae, Eptatretus) from the Pacific Ocean. Proceedings of the California Academy of Sciences 43(16): 249-267.

Meléndez R. 1990. Chironemid fishes from the Juan Fernández Archipelago and Desventuradas Islands (Perciformes: Chironemidae). Revista de Biología Marina 25(2): 83-92.

Meléndez R \& C Villalba. 1992. Nuevos registros y antecedentes para la ictiofauna del Archipiélago de Juan Fernández, Chile. Estudios Oceanológicos 11: 3-29.

Molina GI. 1782. Saggio sulla storia naturale del Chili, del signor Abate Giovanni Ignazio Molina, 367 pp. Stamperia di S. Tommaso d'Aquino, Bologna.

Molina GI. 1810. Saggio sulla storia naturale del Chili, 306 pp. Fratelli Masi, Bologna.

Parin NV. 1991. Fish fauna of the Nazca Ridge and Sala y Gomez submarine ridges, the easternmost outpost of the Indo-West Pacific zoogeographic region. Bulletin of Marine Science 49(3): 671-683.

Peña L. 1987. Consideraciones sobre la fauna de artrópodos terrestres de las islas oceánicas chilenas. In: Castilla JC (ed). Islas oceánicas chilenas: conocimiento científico y necesidades de investigaciones, pp. 217-223. Ediciones Universidad Católica de Chile, Santiago.

Pequeño G. 1989. Peces de Chile. Lista sistemática revisada y comentada. Revista de Biología Marina 24(2): 1-132.

Pequeño G. 1995. Peces. In: Simonetti JA, MTK Arroyo, AE Spottorno \& E Lozada (eds). Diversidad biológica de Chile, pp. 302-313. Comisión Nacional de Investigación Científica y Tecnológica, Santiago.

Pequeño G. 2000. Delimitaciones y relaciones biogeográficas de los peces del Pacífico Suroriental. Estudios Oceanológicos 19: $53-75$.

Pequeño G. 2004. Nemadacylus bergi (Norman, 1937) frente a Bahía Mansa, Chile (Osteichthyes: Cheilodactylidae). Ciencia y Tecnología del Mar 27(1): 121-125.

Pequeño G \& J Lamilla. 1996a. Desventuradas Islands, Chile, the easternmost outpost of the Indo-West Pacific zoogeographic region. Revista de Biología Tropical 44: 929-931.

Pequeño G \& J Lamilla. 1996b. Fishes of the family Serranidae in the Desventuradas Islands, Chile (Osteichthyes, Perciformes). 
Boletin de la Sociedad de Biología de Concepción 67: 22-32.

Pequeño G \& J Lamilla. 2000. The littoral fish assemblage of the Desventuradas Islands (Chile), has zoogeographical affinities with the Western Pacific. Global Ecology \& Biogeography 9: 431-437.

Pequeño G \& S Sáez. 2000. Los peces litorales del archipiélago de Juan Fernández (Chile): endemismo y relaciones ictiogeográficas. Investigaciones Marinas 28: 27-37.

Pequeño G \& S Sáez. 2004. Peces del Crucero CIMAR 6 Islas (II): La Familia Bleniidae en las Islas Desventuradas, Chile (Osteichthyes: Perciformes). Ciencia y Tecnología del Mar 27(1): 113-119.

Pequeño G, A Cea-Egaña \& W Sielfeld. 1992. Primer registro en Chile para tres especies de peces teleósteos marinos, en base a fotografías. Boletín de la Sociedad de Biología de Concepción, Chile 63: 169-173.

Pequeño G, L Vargas \& A Riedemann. 2005. La castañeta Chromis crusma (Valenciennes, 1833) en la costa de Valdivia, con comentarios sobre el género Chromis Cuvier, 1814, en aguas chilenas (Osteichthyes: Pomacentridae). Investigaciones Marinas 33: 101-107.

Pietsch TW \& DB Grobecker. 1987. Frogfishes of the world: Systematics, zoogeography, and behavioral ecology, 420 pp. Stanford University Press, Stanford.

Porter C. 1914. Sur quelques poissons comestibles du Chile et description d'une espece nouvelle. Anales de la Sociedad Científica Argentina 77(304): 185-210.

Randall JE \& JE McCosker. 1975. The eels of Easter Island with a description of a new moray. Contributions in Science, Los Angeles County Museum 264: 1-32.

Randall JE \& R Meléndez. 1987. A new sole of the genus Aseraggodes from Easter Island and Lord Howe Island, with comments on the validity of $A$. ramsaii. Occassional Papers Bernice P. Bishop Museum 27: 97-105.

Randall JE \& A Cea \& R Meléndez. 2005. Checklist of shore and epipelagic fishes of Easter Island, with twelve new records. Boletín. Museo Nacional de Historia Natural, Chile 54: 41-55.

Rendahl H. 1921. The fishes of the Juan Fernandez Islands. In: Skottsberg C (ed). The natural history of Juan Fernandez and Easter Island, 3(1): 49-58. Almqvist \& Wiksells Bocktryckeri, Uppsala.

Richardson J. 1845. Ichthyology of the voyage of H. M. S. Erebus \& Terror under the command of Captain Sir James Clark Ross, R.N, F.R.S. In:. Richardson J \& JE Gray (eds). The zoology of the voyage of H. M. S. "Erebus \& Terror" under the command of Captain Sir James Clark Ross, during the years 1839 to 1843 , 2(2): 1-139. E.W. Janson, London.

Robins CR. 1991. Two new species of Ophidion (Pisces: Ophidiidae) from remote islands of the eastern Pacific. Contributions in Science, Los Angeles County Museum 427: 1-11.
Rojas JR \& G Pequeño. 1998a. Peces serránidos de la isla Alejandro Selkirk, Archipiélago Juan Fernández, Chile (Pisces: Serranidae): Análisis ictiogeográfico. Investigaciones Marinas 26: 41-58.

Rojas JR \& G Pequeño. 1998b. Plectranthias lamillai, a new Anthiine fish species (Perciformes, Serranidae) from the Juan Fernández Archipelago. Scientia Marina 62(3): 203209.

Rojas JR \& G Pequeño. 1998c. Revisión taxonómica de los peces de la subfamilia Anthiinae del Pacífico suroriental chileno (Pisces: Serranidae: Anthiinae). Revista de Biología Marina y Oceanografía 33(2): 163-198.

Rojas JR, S Palma \& G Pequeño. 1998. Food of the grouper Caprodon longimanus from Alejandro Selkirk Island, Chile (Perciformes: Seranidae). Revista de Biología Tropical 46(6): 937-942.

Roszbaczylo N \& JC Castilla. 1987. Invertebrados marinos del Archipiélago de Juan Fernández. In: Castilla JC (ed). Islas oceánicas chilenas: conocimiento científico y necesidades de investigaciones, pp. 167-190. Ediciones Universidad Católica de Chile, Santiago.

Russell BC. 1988. Revision of the labrid fish genus Pseudolabrus and allied genera. Records of the Australian Museum, Supplement 9: 1-72.

Russell BC. 1999. Synodontidae. In: Carpenter KE \& VH Niem (eds). FAO species identification guide for fishery purposes. The living marine resources of the Western Central Pacific, vol. 3: Batoid fishes, chimaeras and bony fishes, Part 1 (Elopidae to Linophrynidae), pp. 1928-1945. FAO, Rome.

Santelices B. 1987. Flora marina bentónica de las islas oceánicas chilenas. In: Castilla JC (ed). Islas oceánicas chilenas: conocimiento científico y necesidades de investigaciones, pp. 101-126. Ediciones Universidad Católica de Chile, Santiago.

Schultz LP. 1944. A revision of the American clingfishes, family Gobiesocidae, with descriptions of new genera and forms. Proceedings of the United States National Museum 96(3187): 47-77.

Schultz LP. 1956. A new pinecone fish, Monocentris reedi, from Chile, a new family record for the eastern Pacific. Proceedings of the United States National Museum 106(3365): 237-239.

Sepúlveda I. 1987. Peces de las islas oceánicas chilenas. In: Castilla JC (ed). Islas oceánicas chilenas: conocimiento científico y necesidades de investigaciones, pp. 225-246. Ediciones Universidad Católica de Chile, Santiago.

Sepúlveda I \& G Pequeño. 1985. Fauna íctica del archipiélago de Juan Fernández. In: Arana P (ed). Investigaciones marinas en el Archipiélago de Juan Fernández, pp. 81-91. Editorial Universitaria, Santiago.

SHOA. 1994. Atlas oceanográfico para la educación, 129 pp. Servicio Hidrográfico y Oceanográfico de la Armada de Chile, Valparaíso. 
Steindachner F. 1875. Ichthyologische Beiträge (II). I. Die fische von Juan Fernandez in den Sammlungen des Wiener Museums. II. Über einige neue Fischarten von der Ost- und Westküste Süd-Amerikas. Sitzungsberichte der Kaiserlichen der Akademie der Wissenschaften 71(1): 443-480.

Steindachner F. 1898. Die fische der Sammlung Plate. In: Fauna Chilensis. Abhandlungen zur Kenntniss der Zoologie Chiles. Zoologische Jahrbuecher Suppl. 4: 281-338.

Steindachner F. 1903. Die fische der Sammlung Plate. (Nachtrag.). Zoologische Jahrbuecher, Jena Suppl. 6: 201214.

Stuessy TF, KA Foland, JF Sutter, RW Sanders \& M Silva. 1984. Botanical and geological significance of potassiumargon dates from Juan Fernández Islands. Science 225: 49-51.
Stuessy TF, C Marticorena, R Rodríguez, DJ Crawford \& M Silva. 1992. Endemism in the vascular flora of the Juan Fernández Islands. Aliso 13(2): 297-307.

Vera R \& G Pequeño. 2001. Comparación de caracteres merísticos y morfométricos entre peces del género Sicyases del archipiélago de Juan Fernández, Valparaíso y Valdivia (Osteichthyes: Gobiesocidae). Investigaciones Marinas 29(2): 3-14.

Walker HJ Jr. \& KW Radford. 1992. Eastern Pacific species of the genus Umbrina (Pisces: Sciaenidae) with a description of a new species. Fishery Bulletin 90: 574-587.

Whitley GP. 1932. Studies in ichthyology. No. 6. Records of the Australian Museum 18(6): 321-348.

Wisner RL. 1966. Cruise report, research vessel Anton Bruun, cruise 12. Marine Laboratory, Texas A\&M University at Galveston, Texas, Special Report 2: 1-14.

Recibido el 17 de noviembre de 2009 y aceptado el 16 de marzo de 2010 